\title{
The Impact of Mountain Waves on an Idealized Baroclinically Unstable Large-Scale Flow
}

\author{
Maximo Q. Menchaca And Dale R. DurRan \\ Department of Atmospheric Sciences, University of Washington, Seattle, Washington
}

(Manuscript received 27 December 2017, in final form 15 July 2018)

\begin{abstract}
The feedback of mountain waves and low-level blocking on an idealized baroclinically unstable wave passing over an isolated ridge is examined through numerical simulation. Theoretical analysis implies that the volumeintegrated perturbation momentum budget is dominated by mean-flow deceleration, the divergence of vertical fluxes of horizontal momentum, and the Coriolis force acting on the perturbation ageostrophic wind. These do indeed appear as the dominant balances in numerically computed budgets averaged over layers containing 1) wave breaking in the lower stratosphere, 2) flow blocking with wave breaking near the surface, and 3) a region of pronounced horizontally averaged mean-flow deceleration in the upper troposphere where there is no wave breaking. The local impact of wave breaking on the jet in the lower stratosphere is dramatic, with winds in the jet core reduced by almost $50 \%$ relative to the no-mountain case. Although it is the layer with the strongest average deceleration, the local patches of decelerated flow are weakest in the upper troposphere. The crossmountain pressure drag over a $2-\mathrm{km}$-high ridge greatly exceeds the vertical momentum flux at mountain-top level because of low-level wave breaking, blocking, and lateral flow diversion. These pressure drags and the low-level momentum fluxes are significantly different from corresponding values computed for simulations with steady forcing matching the instantaneous conditions over the mountain in the evolving large-scale flow.
\end{abstract}

\section{Introduction}

When a stratified airstream crosses a mountain, pressure perturbations may develop along the surface that tend to accelerate the mountain in the direction of airflow. An equal and opposite "pressure drag" is exerted back on the atmosphere by the topography, and gravity waves (mountain waves) are generated that transport momentum downward. In the simplest case, this downward transport occurs between some level subject to "gravity wave drag" where vertically propagating mountain waves dissipate and the surface where the momentum flux balances the pressure drag. That simplest case may be analyzed by linearizing the governing equations, with Coriolis forces neglected, about a steady horizontally uniform flow in which all perturbations are assumed to vanish sufficiently far upstream and downstream of the topography (Eliassen and Palm 1960). The important influence of such gravity wave drag on the larger-scale flow has long been recognized (Sawyer 1959; Lilly 1972; Smith 1979b), and this effect is parameterized in all coarse-resolution weather and

\footnotetext{
Corresponding author: Dale R. Durran,drdee@uw.edu
}

climate models [see Kim et al. (2003) for a review]. A large number of major field programs have attempted to observe mountain waves and their associated momentum fluxes in an ongoing effort to better understand mountain-induced gravity wave drag and improve its parameterization in weather prediction and climate models (Lilly et al. 1982; Davies and Phillips 1985; Bougeault et al. 1997; Smith et al. 2007; Doyle et al. 2011; Fritts et al. 2016). Numerical simulations of cases observed during these field programs have provided increasingly detailed representations of the mountain waves and momentum fluxes for specific real-world conditions (e.g., Kruse et al. 2016), but such case studies are not easily generalizable to describe generic prototypical behaviors.

The pioneering theoretical work by Eliassen and Palm (1960) on momentum transport by vertically propagating mountain waves has been extended in several ways to create a large body of work, much of which is focused on terrain-generated perturbations in steady environmental flows without the inclusion of the Coriolis force $f$. Among those studies that include the Coriolis force, but retain the assumption of a steady large-scale environment, Jones (1967) and Bretherton (1969) examined 
vertical momentum flux profiles in linear waves when $f$ is nonzero, and Ólafsson and Bougeault (1997) and Wells et al. (2005) conducted numerical simulations of flows over isolated three-dimensional ridges. Studies that include temporal variations in the large-scale flow impinging on the mountain include Lott and Teitelbaum (1993a,b), who considered linear mountain waves in a two-dimensional flow with constant basic-state BruntVäisälä frequency $N$ and a time-varying, vertically uniform wind $U(t)$, and Chen et al. (2005), who examined the momentum fluxes transported by finite-amplitude mountain waves triggered by the passage of a large-scale barotropic jet over an isolated 3D ridge.

Relatively few idealized theoretical studies have focused on the large-scale response generated by breaking mountain waves. Nonlinear 2D numerical simulations by Durran (1995) demonstrated that, at least in the absence of Coriolis forces, the flow deceleration that develops in response to wave breaking can take the form of very small perturbations spread over a very large horizontal domain. When Coriolis forces are present, one might expect geostrophic adjustment to impose an additional constraint on the scales affected by the decelerative forcing. Chen et al. (2007), expanding on Chen et al. (2005), found that wave breaking produced potential vorticity (PV) anomalies that gradually organized into large-scale structures downstream from the mountain. These PV anomalies were associated with quasigeostrophically balanced perturbation velocities at the wave breaking level, which reduced a $20 \mathrm{~m} \mathrm{~s}^{-1}$ jet maximum by $5 \mathrm{~m} \mathrm{~s}^{-1}$.

Because Chen et al. (2007) considered a barotropic jet, in which $N$ and $U$ were constant with height at any given $x, y$, and $t$, wave breaking occurred at the low altitude of $3 \mathrm{~km}$, whereas in typical real-world flows, an important wave-breaking region develops in the lower stratosphere in response to increases in $N$ and decreases in $U$ with height (e.g., Lilly and Kennedy 1973). The goal of this paper is to examine the upscale influence of terrain-induced perturbations in a prototypical midlatitude system. We address this problem using numerical simulations of the same large-scale environment interacting with the same topography considered in Menchaca and Durran (2017, hereafter MD17), which consists of an idealized midlatitude cyclone growing in a baroclinically unstable flow that encounters an isolated $3 \mathrm{D}$ ridge. In the following we report on the momentum fluxes and mean-flow perturbations that develop in association with the waves and low-level blocking triggered by that ridge.

Those details of the numerical simulations that differ from MD17 are summarized in section 2. The relationship between the cross-mountain pressure drag and the lower-tropospheric momentum fluxes is presented in section 3. Section 4 examines the momentum fluxes and the horizontally averaged flow response for the 2-km-mountain case, while the local large-scale flow response for this case is covered in section 5. Section 6 contains the conclusions.

\section{Simulation details and overview}

The large-scale flow and the initiation of the cyclone are described in MD17, along with the shape of the isolated ridge, whose approximate $x$ and $y$ extents are 80 and $640 \mathrm{~km}$, respectively. The same two mountain heights are again considered: $500 \mathrm{~m}$ and $2 \mathrm{~km}$. The 500-m-mountain simulations are exactly those described in MD17, but the 2-km-mountain simulations, while very similar, were repeated using slightly higher resolution and a much larger nested grid to better capture the small-scale PV anomalies near the mountain and their interaction with large scales well downstream (the nesting is one way). The difference between the numerical parameters for the two different mountain heights is therefore, that the 500-m-mountain simulation uses $\Delta x=\Delta y=15 \mathrm{~km}$ on the outer grid and $\Delta x=$ $\Delta y=5 \mathrm{~km}$ on the nest, which extends over the region $-380 \leq x \leq 1165 \mathrm{~km},-1125 \leq y \leq 750 \mathrm{~km}$, whereas the 2-km-mountain simulation uses $\Delta x=\Delta y=12 \mathrm{~km}$ on the outer grid and $\Delta x=\Delta y=4 \mathrm{~km}$ on the nest, which covers the region $-372 \leq x \leq 2868 \mathrm{~km},-1128 \leq y \leq 2244 \mathrm{~km}$. In these new 2-km simulations, the surface roughness was also increased to $0.1 \mathrm{~m}$ and the boundary layer height was set at model level 6 , roughly at a height of $282 \mathrm{~m}$. As in the coarser-resolution 2-km-mountain simulations in MD17, the damping layer begins at $14.5 \mathrm{~km}$ and is $6 \mathrm{~km}$ deep, ${ }^{1}$ and there are 80 vertical levels spaced at $30 \mathrm{~m}$ near the surface, with $\Delta z$ increasing to $400 \mathrm{~m}$ near the model top. For each simulation with a mountain, a second no-mountain simulation was conducted using identical model parameters (grid spacing, nest size, etc.), except that the surface was flat.

An overview of the evolution of the large-scale flow is provided in Fig. 1, which shows surface isobars and the height of the 500-hPa surface at 1-day intervals beginning 4.5 days after an isolated PV anomaly was introduced to a baroclinically unstable zonal jet at the start of the simulation. At 4.5 days, the surface cold front has

\footnotetext{
${ }^{1}$ There is little sensitivity to the height of the damping layer in the 2-km-mountain simulation because the mountain is high enough to produce vigorous wave breaking below the damping layer. There is much more sensitivity in the $500-\mathrm{m}$ case, and in that case, the base of the damping layer was set at $20.5 \mathrm{~km}$. See MD17 for further discussion.
} 

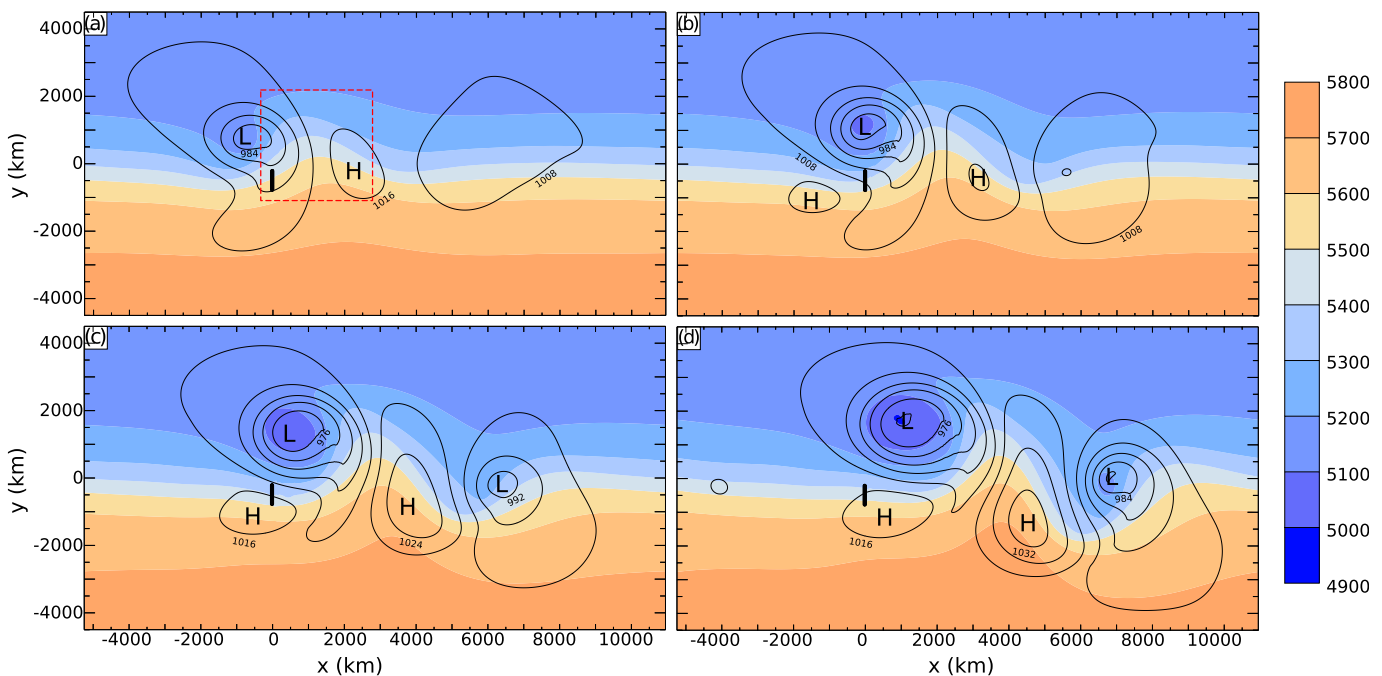

FIG. 1. Surface isobars (black lines at 8 -hPa intervals) and 500-hPa height (color fill at $100-\mathrm{m}$ intervals) for the developing cyclone at (a) 4.5, (b) 5.5, (c) 6.5, and (d) 7.5 days. The mountain is depicted by the black vertical bar at $x=0 \mathrm{~km}$ in all panels. The nested grid for the $2-\mathrm{km}$-mountain simulation is shown in by the red dashed square in (a). Lows and highs are labeled by an $\mathrm{L}$ and $\mathrm{H}$, respectively.

almost reached the mountain (indicated by the black vertical bar). The cyclone continues to deepen, and the upper-level wave continues to amplify throughout the remainder of the simulation, although the crossmountain flow gradually becomes more zonal as the low center translates downstream of the ridge. The mountain-wave response generated by this flow across the $2-\mathrm{km}$ ridge is shown in an east-west vertical cross section along the centerline of the ridge in Fig. 2 for the same four times shown in Fig. 1. Significant wave activity starts to develop just before the cold front strikes the mountain at 4.5 days, although wave breaking has not
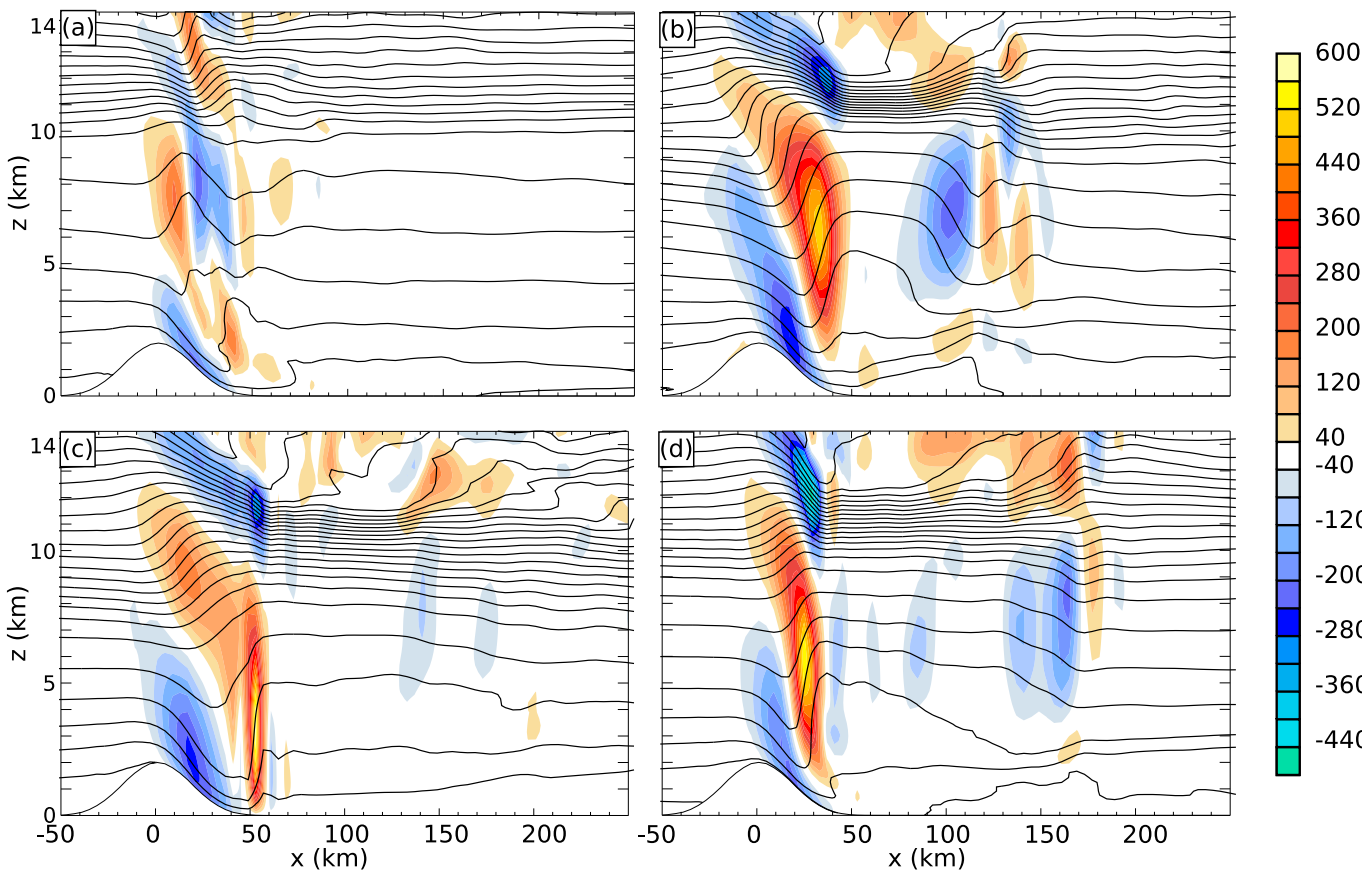

40

FIG. 2. East-west vertical cross sections of $w$ (colors; 40 -cm intervals) and $\theta$ (contours; 5 -K intervals) across the centerline of the mountain at (a) 4.5 , (b) 5.5 , (c) 6.5 and (d) 7.5 days. 
yet begun in the stratosphere (Fig. 2a). The mountain waves remain well developed through the remainder of the simulation, with stratospheric wave breaking present at 5.5, 6.5, and 7.5 days, as evident from the regions of low static stability with almost vertically oriented isentropes. At all times there is some degree of low-level blocking upstream of the ridge. The complex evolution of the waves and the blocked flow is discussed in detail in MD17.

\section{Pressure drag and lower-tropospheric momentum fluxes}

In this section we examine the evolution of the pressure drag and its relation to the vertical momentum flux at levels slightly above the mountain. We will also compare the behavior for the full evolving flow with waves generated by steady forcing in $3 \mathrm{D}$ simulations representative of the large-scale environment at days 4.5-7.5.

The ridge-perpendicular $(x)$ component of the total pressure drag exerted on the atmosphere by the topography within the subdomain $\left[x_{1}, x_{2}\right] \times\left[y_{1}, y_{2}\right]$ may be written as

$$
P(t)=-\int_{x_{1}}^{x_{2}} \int_{y_{1}}^{y_{2}} p[x, y, h(x, y), t] \frac{\partial h}{\partial x} d y d x .
$$

Even if no waves are present, $P$ will include contributions from horizontal variations in the synoptic-scale pressure field. Letting primed variables denote the difference between a field in the simulation with terrain and the corresponding field in the simulation without terrain, the mountain-induced component of the pressure drag is

$$
P^{\prime}(t)=-\int_{x_{1}}^{x_{2}} \int_{y_{1}}^{y_{2}} p^{\prime}[x, y, h(x, y), t] \frac{\partial h}{\partial x} d y d x .
$$

Like $p^{\prime}$, the terrain-induced velocities in our simulation will be defined as the difference between the fields in corresponding pairs of simulations with and without mountains. In our analysis, the vertical flux of the terrain-induced $x$-component momentum within the subdomain $\left[x_{1}, x_{2}\right] \times\left[y_{1}, y_{2}\right]$ is computed as

$$
M^{\prime}(z, t)=\int_{x_{1}}^{x_{2}} \int_{y_{1}}^{y_{2}} \rho u^{\prime} w^{\prime} d y d x
$$

For all analyses in this section, $\left(x_{1}, x_{2}\right)=(-372,1145) \mathrm{km}$, $\left(y_{1}, y_{2}\right)=(-1128,172) \mathrm{km}$, which gives the maximum sized box having the terrain centered in the north-south direction that fits within the fine-nest subdomain used in the 500-m-high-mountain simulation.

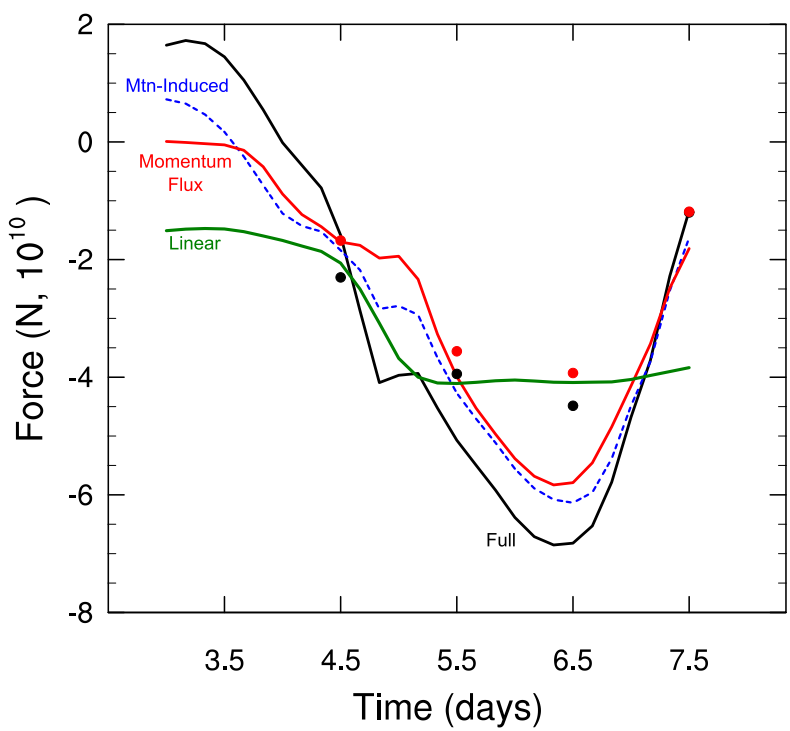

FIG. 3. Pressure drags and momentum fluxes plotted as function of time for the 500-m-mountain simulations: full pressure drag $P$ (solid black), mountain-induced pressure drag $P^{\prime}$ (dashed blue), linear steady-state estimate of the drag $P_{\text {lin }}$ (green solid), and simulated momentum flux $M^{\prime}$ a $z=550 \mathrm{~m}$ (red). Also shown are the pressure drags and momentum fluxes (black and red dots, respectively) from steady 3D simulations forced by conditions representative of those over the mountain at $4.5,5.5,6.5$, and 7.5 days. (Red and black dots almost coincide at 7.5 days.)

\section{a. 500-m-high mountain}

The full pressure drag $P$, the mountain-induced component $P^{\prime}$, and the momentum flux $M^{\prime}$ across a horizontal plane $50 \mathrm{~m}$ above mountain top are compared in Fig. 3 for the 500-m ridge. The full pressure drag $P$ from the evolving simulation (solid black line) is positive early in the simulation when the low pressure center is northwest of the ridge. As the cyclone propagates zonally, the pressure drag quickly becomes negative and increases in magnitude as the cold front impinges on the terrain, reaching an extremum of almost $-7 \times 10^{10} \mathrm{~N}$ at 6.4 days before rapidly decreasing in magnitude during the remainder of the simulation. The mountain-induced pressure drag $P^{\prime}$ (dashed blue line) shows a similar evolution, but is weaker in magnitude than $P$, achieving values of about $85 \%$ of the full pressure drag at 6.4 days. Although the synoptic-scale contribution $P-P^{\prime}$ is not completely negligible, the mountain-induced component is clearly dominant. Neglecting the Coriolis force, linear theory for the steady inviscid mountainwave problem implies the pressure drag at the surface equals the vertical momentum flux. The 500-m-high mountain does not generate a highly nonlinear response, 
and as evident in Fig. 3, the mountain-induced drag is approximately equal to the momentum flux. ${ }^{2}$

Simple estimates of the cross-mountain pressure drag are potentially useful for parameterizing the subgridscale influences of mountain waves and topographic forcing. Bessemoulin et al. (1993) found that the linear pressure drag for steady 2D flow of uniform speed $U$ and Brunt-Väisälä frequency $N$ over a Witch of Agnesi mountain of height $h_{0}$ in the hydrostatic nonrotating limit,

$$
P_{\text {lin }}=-\frac{\pi}{4} \rho U N h_{0}^{2}
$$

gave a good estimate of the observed pressure drag per unit distance along the Pyrenees ridge during the Momentum Budget over the Pyrenees (PYREX) Experiment. The cross-mountain width of the Pyrenees is similar to the roughly $80-\mathrm{km}$ width of our ridge, and they are a larger amplitude obstacle, with many peaks higher than $3 \mathrm{~km}$. Thus, on the basis of Bessemoulin et al. (1993), one might expect that $P_{\text {lin }}$ would give a good estimate of the simulated mountain-induced drag over our 500-m-high ridge. We computed $P_{\text {lin }}$ (green line in Fig. 3) using values of $U$ and $N$ obtained from vertical averages between the surface and the tropopause ${ }^{3}$ at a point $100 \mathrm{~km}$ upstream of the center of the ridge. In contrast to the results in Bessemoulin et al. (1993), this simple estimate of the pressure drag gives a poor approximation of the mountain-induced drag over most of the 4-day period shown in Fig. 3. In particular, $P_{\text {lin }}$ gives substantially more drag than $P^{\prime}$ at 3.5 days, does a fairly good job between 4 and 5.5 days, and then completely fails to track the subsequent variations in the mountaininduced drag by remaining roughly constant for the last 2 days, during which time $P^{\prime}$ rapidly drops to, and rises from, a negative extremum at 6.4 days. The errors in $P_{\text {lin }}$ may be due to the transient nature of the flow, the crudeness with which (4) approximates the true linear solution in the presence of spatially nonuniform crossmountain winds and static stabilities, or to nonlinearity.

Errors arising from the steady-state assumption can be isolated by analyzing steady-state 3D simulations for a flow over an identical ridge forced by steady upstream conditions matching the large-scale $u$ and $\theta$ in

\footnotetext{
${ }^{2}$ The terms $M^{\prime}$ and $P^{\prime}$ would also be equal in the steady inviscid finite-amplitude case if 1 ) the flow at the surface is parallel to the topography, and 2) the horizontal momentum fluxes far from the mountain through vertical sidewalls, extending from the surface to a height of $50 \mathrm{~m}$ above mountain top, are unperturbed (i.e., the disturbance at low levels vanishes far away from the mountain).

${ }^{3}$ The value of $\rho$ for $P_{\text {lin }}$ is the vertical average between the surface and twice the mountain height.
}

a $y-z$ vertical plane $200 \mathrm{~km}$ upwind of the ridge. These steady 3D simulations were conducted in a domain identical to the fine-mesh subdomain in the evolvingflow simulation for conditions corresponding to days 4.5, 5.5, 6.5, and 7.5. ${ }^{4}$ The steady-state pressure $\operatorname{drag} P_{\mathrm{ss}}$ is the time-mean value from the final $5 \mathrm{~h}$ of each $24-\mathrm{h}$ simulation, by which period the pressure drag has become almost steady. Plotted as a black dot in Fig. 3 for each of the four simulation times, $P_{\mathrm{ss}}$ agrees well with both $P^{\prime}$ and $P_{\text {lin }}$ at 4.5 and 5.5 days. At 6.5 days, $P_{\text {ss }}$ roughly agrees with $P_{\text {lin }}$, but not $P^{\prime}$, suggesting that flow transience plays a significant role in generating the large extremum in the terrain-induced pressure drag in the full simulation. At 7.5 days, $P_{\mathrm{ss}}$ agrees with $P^{\prime}$, but not $P_{\text {lin }}$, suggesting that the error in $P_{\text {lin }}$ arises from an overly crude representation of the actual structure of the background flow, or perhaps nonlinearity.

To estimate the potential impact of nonlinearity, we again use values of $N$ and $U$ averaged through the depth of the troposphere to evaluate the nonlinearity parameter $N h_{0} / U$. At 4.5 days, $N h_{0} / U=0.29$, and at 7.5 days it is 0.24 . In a true constant- $N$ and constant- $U$ atmosphere, the surface pressure drag for such values of $N h_{0} / U$ would be only about $10 \%$ higher than that predicted by linear theory (Epifanio and Durran 2001, their Fig. 3). But when there are abrupt variations in the static stability, such as a factor-of-2 change at the tropopause, the deviations from the drag predicted by linear theory can easily exceed $50 \%$ when $N h_{0} / U=0.25$ (Durran 1992, his Fig. 7). Nonlinear effects are, therefore, a possible cause of the differences between $P^{\prime}$ and $P_{\text {lin }}$.

The momentum flux $50 \mathrm{~m}$ above mountain-top level $M_{\mathrm{ss}}$ was also computed for the four steady-state simulations. Those values are plotted as red dots in Fig. 3 . The agreement between $M_{\mathrm{ss}}$ and $P_{\mathrm{ss}}$ is reasonably good and similar to that between $P^{\prime}$ and $M^{\prime}$ for the evolving flow.

\section{b. 2-km-high mountain}

Figure 4 shows the results of a similar analysis for the 2-km-high mountain. The increase in mountain height makes the mountain-induced drag much larger than the synoptic-scale component, so that $P^{\prime}$ agrees with $P$ much better than in the 500-m case. If the upstream $U$ and $N$ are unchanged, (4) implies the factor of 4 increase in the mountain height will increase the magnitude of the linear drag by a factor of 16 without substantially modifying its temporal variation. The shapes of the $P_{\text {lin }}^{\prime}$

\footnotetext{
${ }^{4}$ The initialization procedure for the steady-flow simulations is described in detail in section $4 \mathrm{~b}$ from MD17.
} 


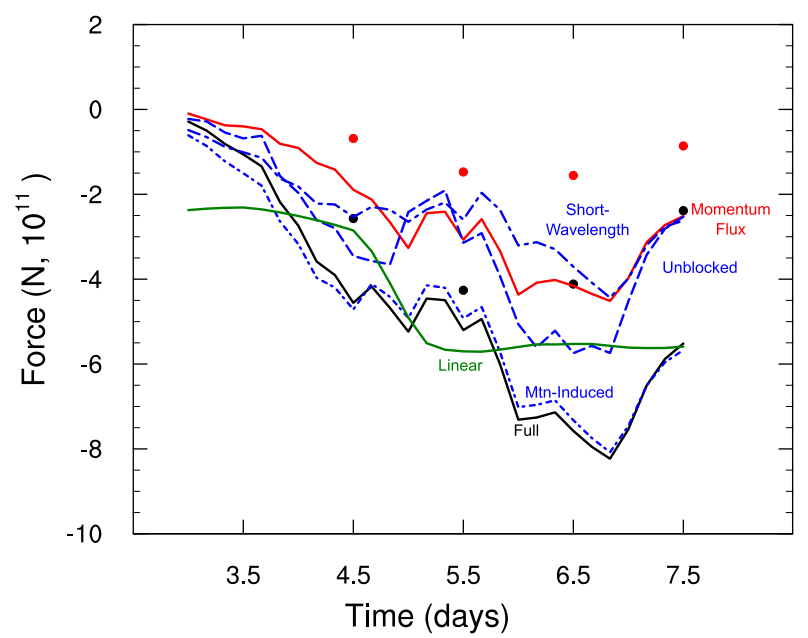

FIG. 4. As in Fig. 3, but for the 2-km mountain. The momentum fluxes are now computed at $z=2050 \mathrm{~m}$. Also plotted are the shortwavelength pressure $\operatorname{drag} P_{\mathrm{sw}}^{\prime}$ (dot-dashed blue) and the unblocked pressure drag $P_{\text {ub }}^{\prime}$ (long dashed blue).

curves in Figs. 3 and 4 are indeed similar, although small changes in $U$ and $N$ resulting from upstream influences from the higher topography reduce the increase in $P_{\text {lin }}^{\prime}$ to about a factor of 14 . As in the $500-\mathrm{m}$ case, the agreement between $P^{\prime}$ and $P_{\text {lin }}^{\prime}$ is very poor.

In contrast to the 500-m case, the momentum flux (now calculated at $z=2050 \mathrm{~m}$ ) is much smaller than $P^{\prime}$. For example, the negative extremum in $M^{\prime}$ at 6.8 days is about half the magnitude of the extremum in $P^{\prime}$. A similar factor-of- 2 difference between the simulated pressure drag and the low-level momentum flux averaged over the entire Pyrenees mountains was obtained in a case study from the PYREX (Bougeault et al. 1993, their Fig. 22). Why is the momentum flux so much smaller than the mountaininduced drag in our simulations? Linear theory suggests that the wave drag and momentum fluxes associated with a relatively wide sinusoidal mountain are reduced as $f / U k$ approaches one from below; here $k$ is the wavenumber and $f$ is the Coriolis parameter. ${ }^{5}$ The pressure drag in the $f \neq 0$ hydrostatic case for uniform flow across sinusoidal ridges with amplitude $h_{0} / 2$ is

$$
P_{\text {lf }}=-\frac{\pi}{4} \rho U N h_{0}^{2}\left(1-\frac{f^{2}}{U^{2} k^{2}}\right)^{1 / 2} .
$$

When Coriolis effects are not negligible, the momentum flux does not equal $P_{\mathrm{lf}}$; instead

\footnotetext{
${ }^{5}$ Smith (1979a) and Gill (1982, his Fig. 8.10) give the behavior as a function of mountain width for a Witch of Agnesi mountain.
}

$$
P_{\mathrm{lf}}=\int_{0}^{2 \pi / k} \rho\left(u^{\prime}-f \eta^{\prime}\right) w^{\prime} d x
$$

where $\eta^{\prime}$ is the displacement parallel to the $y$ coordinate produced by the perturbation $y$-component velocities (Jones 1967; Bretherton 1969). In the more linear 500-m-mountain case, Coriolis effects are sufficiently small that $P^{\prime}$ provides a good estimate of $M^{\prime}$, implying that the Rossby number associated with the environmental cross-mountain flow is large enough to make the $f \eta^{\prime} w^{\prime}$ term in (6) insignificant. Nonlinear processes are therefore the likely source of the difference between $P^{\prime}$ and $M^{\prime}$ in the 2-km-mountain case. Figure 2 suggests that wave breaking below $2 \mathrm{~km}$ contributes to the reduction of $M^{\prime}$ relative to $P^{\prime}$. In addition, low-level blocking associated with flow splitting and lateral diversion around the ends of the ridge may contribute to the pressure drag without efficiently generating vertically propagating waves, thereby further reducing $M^{\prime}$ relative to $P^{\prime}$. Since lateral diversion and low-level breaking can both create wakes that decay slowly downstream, the agreement between the pressure drag and momentum flux might be better if we focus only on the short-wavelength contributions to $P^{\prime}$.

The short-wavelength contributions to the mountaininduced surface pressure drag were therefore estimated in the following manner. First, a horizontally uniform hydrostatically balanced reference profile was subtracted from the total pressure at the surface to obtain the field $\tilde{p}(x, y, t)$ in the $2-\mathrm{km}$-mountain simulation. This procedure removes the signal in the surface pressure field associated with changes in topographic height. After calculating the analogous $\tilde{p}(x, y, t)$ at the corresponding heights in the no-mountain simulation, the two fields were differenced to obtain $\tilde{p}^{\prime}(x, y, t)$. The short-wavelength contributions to surface pressure perturbation $p_{\mathrm{sw}}^{\prime}(x, y, t)$ were evaluated by applying an eighth-order Butterworth filter to $\tilde{p}^{\prime}(x, y, t)$ with a cutoff wavelength parallel to the $x$ axis of $\lambda_{x}=200 \mathrm{~km}$. Finally, the short-wavelength mountain-induced pressure drag was computed as

$$
P_{\mathrm{sw}}^{\prime}(t)=-\int_{x_{1}}^{x_{2}} \int_{y_{1}}^{y_{2}} p_{\mathrm{sw}}^{\prime}(x, y, t) \frac{\partial h}{\partial x} d y d x .
$$

Before examining the short-wavelength pressure drag, it is helpful to consider the distribution of total, short-wavelength, and long-wavelength surface pressures relative to the mountain. Figure 5 shows $p_{\mathrm{sw}}^{\prime}$, along with the total mountain-induced pressure at the height of the topography $p^{\prime}[x, y, h(x, y)]$, and their difference at 6.5 days into the 500-m-high-mountain simulation. The $p^{\prime}$ field shows a clear signature of high pressure on 

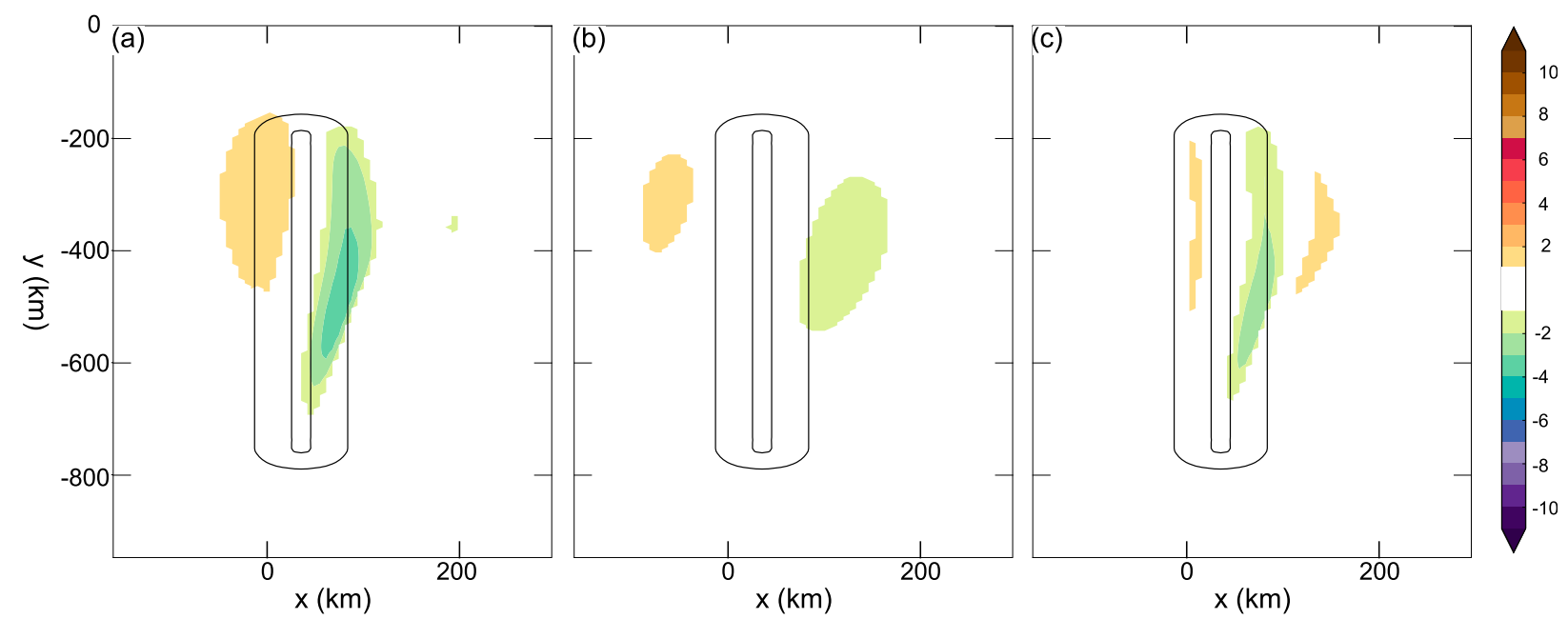

FIG. 5. Surface pressure perturbations induced by the 500-m-high mountain at $t=6.5$ days: (a) total $p^{\prime}[x, y, h(x, y)]$, (b) large-scale contribution $p^{\prime}[x, y, h(x, y)]-p_{\mathrm{sw}}^{\prime}$, and (c) short-wavelength contributions $p_{\mathrm{sw}}^{\prime}$. Contour interval is 1 -hPa, terrain is contoured at 50 and $450 \mathrm{~m}$. The axes are stretched along the $x$ coordinate for visibility.

the upstream slopes, with low pressure on the lee (Fig. 5a), and as apparent in Fig. 5c, along the slopes of the topography, the dominant contribution to $p^{\prime}$ is provided by $p_{\mathrm{sw}}^{\prime}$. The long-wavelength components of the mountain-induced pressure field $p^{\prime}[x, y, h(x, y)]-p_{\mathrm{sw}}^{\prime}$ are maximized over flat terrain, upstream and downstream of the ridge, where they make no contribution to the pressure drag (Fig. 5b). Like the extrema in $p^{\prime}$ itself, those in $p^{\prime}[x, y, h(x, y)]-p_{\mathrm{sw}}^{\prime}$ are shifted north and rotated clockwise with respect to the center of the ridge.

The $p^{\prime}[x, y, h(x, y)]$ field for the 2-km-mountain case is plotted along with $p_{\mathrm{sw}}^{\prime}$, and their difference at $t=6.5$ days in Fig. 6. In the $2-\mathrm{km}$ case, the mountain again produces a large region of negative pressure anomalies along the lee slope, and the distribution of the $p_{\mathrm{sw}}^{\prime}$ field is similar to, though 2.5 times larger in amplitude than, that in the 500-m case (Fig. 6c). But in contrast to the $500-\mathrm{m}$ case, there is a pronounced large-scale signal in the perturbation pressure field with positive values in the north and negative values in the south (Figs. 6a,b). Much stronger large-scale pressure perturbations are now also present over the ridge itself, accounting for the significant difference between $P^{\prime}$ and $P_{\mathrm{sw}}^{\prime}$ in the $2-\mathrm{km}$-mountain case evident in Fig. 4.

The pattern of $p^{\prime}[x, y, h(x, y)]$, with positive values in the north and negative values in the south, is grossly
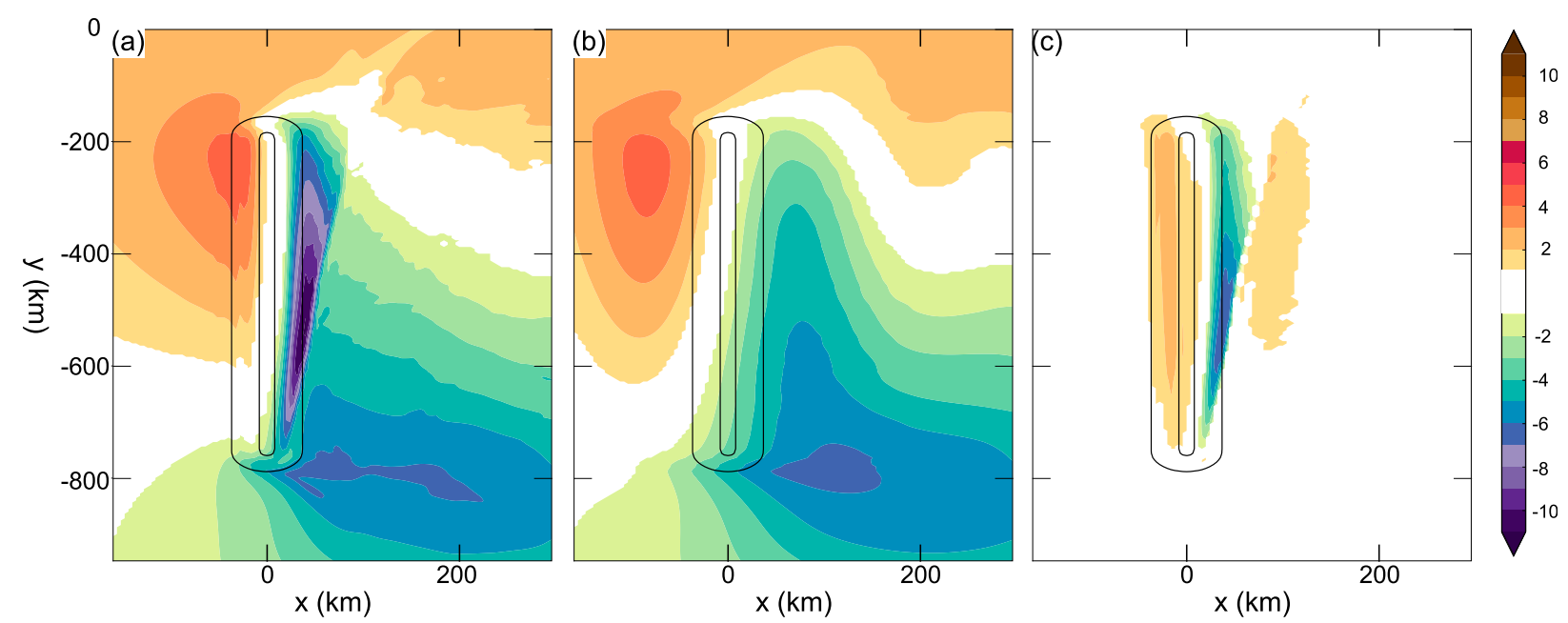

FIG. 6. As in Fig. 5, but for the 2-km mountain. The terrain is contoured at $200 \mathrm{~m}$ and $1.8 \mathrm{~km}$. 
similar to that obtained by Wells et al. (2005) for a more nonlinear case with constant $N$ and $U$. Both our Fig. 6a and Fig. 8b of Wells et al. (2005) also show a positive extremum on the windward slopes near the north end of the mountain and a negative extremum well downstream of the south end of the ridge. The intense trough of wave-induced low pressure along the lee slopes is not, however, apparent in Fig. 8b of Wells et al. (2005).

Returning to the relationship between pressure drag and the momentum flux, in the 500-m-mountain case, $P_{\text {sw }}$ agrees very well with the both $P^{\prime}$ and $M^{\prime}$ (not shown). In the 2-km-mountain case, $P_{\mathrm{sw}}$ (dot-dashed blue line in Fig. 4) is a much better approximation to $M^{\prime}$ than is the full $P^{\prime}$. Prior to 4.5 days, $P_{\mathrm{sw}}^{\prime}$ exceeds $M^{\prime}$ by as much as a factor of 2 . After 4.5 days, as the mountain waves strengthen, $M^{\prime}$ modestly exceeds or roughly approximates $P_{\mathrm{sw}}^{\prime}$. What physical processes contribute to the difference between $P^{\prime}$ and $P_{\mathrm{sw}}^{\prime}$ ?

As previously noted for simulations of flow over high ridges by Ólafsson and Bougeault (1997) and Wells et al. (2005), the pressure drag on the 2-km-high mountain is created by two different processes: by the blocking and diversion of the low-level flow around the ends of the ridge (MD17, see their Fig. 19c) and by a strong mountain wave in the overlying crossmountain flow (Fig. 2c). To estimate the relative contributions of each of these processes to the total pressure drag, the drag caused by the unblocked flow was evaluated using the concept of the "dividing streamline" (Snyder et al. 1985), which separates the low-level flow that passes around an obstacle from the flow that passes over the obstacle. We attribute the pressure drag caused by blocking to the flow below the dividing streamline and suppose the mountain-waveinduced pressure drag is contributed by the flow above the dividing streamline.

On the upstream side of the ridge, we estimated the top of the diverted flow (i.e., the height of the dividing streamline) $h_{b}(y)$ at each point along the ridge as the elevation at which the cross-mountain velocity component upstream of the crest drops below $1 \mathrm{~m} \mathrm{~s}^{-1}$. The threshold for flow diversion is set slightly larger than zero because, as noted by Ólafsson and Bougeault (1997), the zones of flow stagnation and reversal that are easily identified in free-slip flow disappear in the presence of surface friction. We estimated the leeside limit of the contribution to the pressure drag from the mountain wave in flow above the dividing streamline as occurring at the height $h_{l}(y)$ on the lee slope below the wave trough, where $\partial p^{\prime} / \partial x$ changes sign. This choice is motivated by an alternate expression for surface pressure obtained using integration by parts:

$$
\begin{aligned}
-\int_{x_{1}}^{x_{2}} p^{\prime} \frac{\partial h}{\partial x} d x= & -p^{\prime}\left(x_{2}\right) h\left(x_{2}\right)+p^{\prime}\left(x_{1}\right) h\left(x_{1}\right) \\
& +\int_{x_{1}}^{x_{2}} h \frac{\partial p^{\prime}}{\partial x} d x
\end{aligned}
$$

Note that the first two terms on the right-hand side are zero if the terrain drops to zero at $x_{1}$ upstream and at $x_{2}$ downstream of the ridge, and that since $\partial p^{\prime} / \partial x$ is zero at the $x$ location where $h(x, y)=h_{l}(y)$, the sensitivity of the unblocked pressure drag to this choice of the downstream limit is at a local minimum with respect to $x$.

The field of unblocked surface pressure was therefore evaluated as

$p_{\mathrm{ub}}^{\prime}(x, y)= \begin{cases}p^{\prime}(x, y, h), & h(x, y)>h_{b}(y) \text { and } x \leq x_{\text {crest }} \\ p^{\prime}(x, y, h), & h(x, y)>h_{l}(y) \text { and } x>x_{\text {crest }} \\ 0, & \text { otherwise }\end{cases}$

and the unblocked pressure drag $P_{\mathrm{ub}}^{\prime}$ was calculated using the integral in (7) with $p_{\mathrm{sw}}^{\prime}$ replaced by $p_{\mathrm{ub}}^{\prime}$. The unblocked pressure drag, which is shown as the long dashed line in Fig. 4, is generally larger than the blocked contribution $\left(P^{\prime}-P_{\mathrm{ub}}^{\prime}\right)$ to the total drag. Around 6.5 days, when the drag is strongest, the unblocked contribution is roughly $75 \%$ of the total drag. Nevertheless, there are periods around 5.5 and 7.5 days when the unblocked contribution is slightly less than half of the total pressure drag. Figure $19 \mathrm{~b}$ in MD17 suggests that the relative contribution of the mountain wave to the total drag may be less around 5.5 days because strong waves are restricted to the northern half of the ridge. At 7.5 days, the waves in the cross-mountain flow extend along the full north-south extent of the ridge, but are weaker than at 6.5 days (Figs. 2c,d), while strong southerly flow as developed in the blocked layer upstream of the ridge (MD17, see their Fig. 19c).

The unblocked pressure drag gives a worse approximation than $P_{\mathrm{sw}}^{\prime}$ to the momentum flux. In particular $P_{\mathrm{ub}}^{\prime}$ is larger than both $P_{\mathrm{sw}}^{\prime}$ and $M^{\prime}$ except for a brief period around 5 days. This could be explained by low-level wave breaking, which would lead to enhanced mountain-wave-induced drag, but leaves a longwavelength wake downstream that would not project onto the short-wavelength pressure drag. In summary, the full mountain-induced pressure drag $P^{\prime}$ exceeds the momentum flux $M^{\prime}$ for two reasons: 1) flow blocking and 2) wave breaking near and below mountain top level. Conceptually, the extent to which $\left|M^{\prime}\right|$ is reduced from $\left|P^{\prime}\right|$ by blocking can be roughly approximated as 
$\max \left(0,\left|P^{\prime}-P_{\mathrm{ub}}^{\prime}\right|\right)$, and the extent to which this difference is further reduced by low-level wave breaking can be crudely approximated as $\max \left(0,\left|P_{\mathrm{ub}}^{\prime}-P_{\mathrm{sw}}^{\prime}\right|\right)$.

The steady-forcing runs, again initialized with synoptic conditions representative of the large-scale crossmountain flow, produce much smaller pressure drags and momentum fluxes (black and red dots in Fig. 4) than the evolving-flow simulation. The differences between the evolving-flow and the steady-forcing runs are much greater when the mountain height is increased from $500 \mathrm{~m}$ to $2 \mathrm{~km}$, suggesting that nonlinear processes enhance the influence of nonsteady forcing on the pressure drag and momentum fluxes. The differences between the pressure drag and the momentum flux in each of the four steady-forcing runs is also much greater for the 2-km-mountain than for the 500-m case, suggesting that nonlinear processes like flow blocking and low-level wave breaking again are playing important roles in the higher-mountain simulations.

\section{Momentum flux and horizontally averaged flow response: $2-\mathrm{km}$-mountain case}

\section{a. Vertical distribution of momentum fluxes and flow deceleration}

Because the influence of the 2-km-high mountain on the large-scale flow is much greater than that of the 500-m mountain, we will focus on the impact of the larger mountain throughout the remainder of this paper. The vertical divergence of the momentum flux exerts a force on the large-scale cross-mountain flow. Values of $M^{\prime}$ are plotted as a function of $z$ and $t$ for the 2-km-high-mountain simulation in Fig. $7 .^{6}$ Here and throughout the remainder of the paper, horizontal averages are computed over the entire 4-km fine mesh, in which case $\left(x_{1}, x_{2}\right)=(-372,2868) \mathrm{km}$ and $\left(y_{1}, y_{2}\right)=$ $(-1128,2244) \mathrm{km}$ in (3). Large low-level vertical gradients in $M^{\prime}$ develop as a consequence of low-level wave breaking around 4 days, well before frontal passage (MD17, their Fig. 13). The fluxes and low-level vertical gradients strengthen up to day 6.8 and then gradually decrease throughout the remainder of the simulation. Care should nevertheless be exercised when interpreting momentum flux gradients below mountain-top level because those values include the pressure drag on the portion of the topography extending above that level,

\footnotetext{
${ }^{6}$ In Fig. 7, momentum flux values at an elevation below the maximum ridge height (say $z_{l}$ ) were computed by integrating $\rho_{0} u^{\prime} w^{\prime}$ at level $z_{l}$ over a horizontal domain excluding the region pierced by the mountain and adding the terrain-induced pressure drag caused by the portion of the ridge with height greater than $z_{l}$.
}

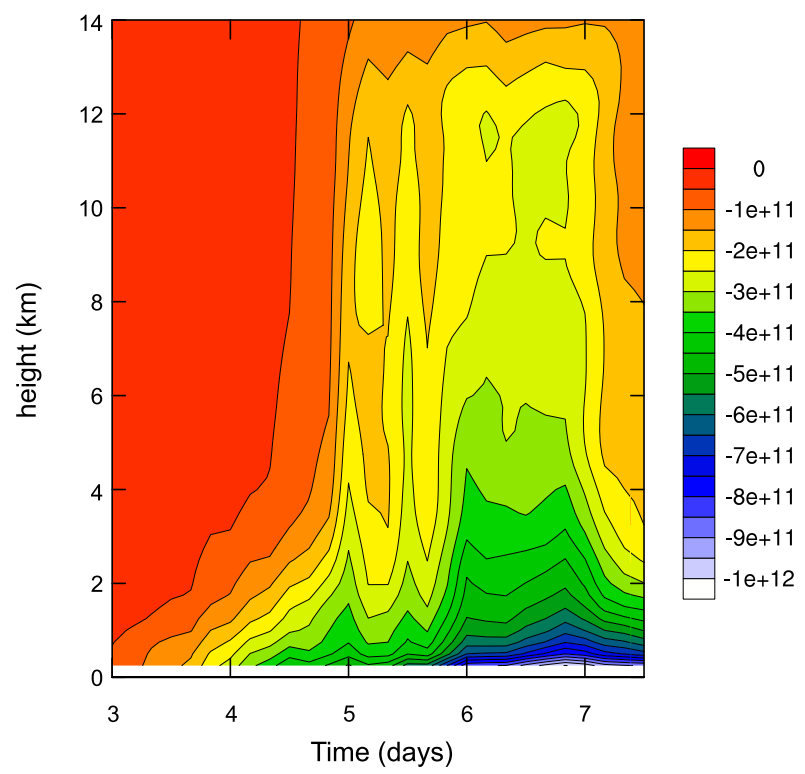

FIG. 7. The momentum flux $M^{\prime}(\mathrm{N})$ in the 2-km-high-mountain simulation as a function of $z$ and $t$, horizontally averaged over the entire nested mesh. At heights $z_{l}$ below the top of the mountain, the mountain-induced pressure drag caused by the portion of the ridge extending above $z_{l}$ is added to the flux in the free air (see text).

and as emphasized in connection with Fig. 4, the terraininduced pressure drag is much larger than the vertical momentum flux.

Wave breaking aloft, in the layer $12 \leq z \leq 14 \mathrm{~km}$, begins around 5 days and continues through 7 days, creating a second region of vertical momentum flux divergence near the top of the domain. The horizontally averaged values of $u^{\prime}$ that develop in response to the vertical divergence of $M^{\prime}$ are plotted as a function of $z$ and $t$ in Fig. 8. For a given vertical momentum flux divergence, stronger deceleration occurs aloft because of the decrease in density with height. Before frontal passage at 4.5-5 days, the velocity perturbations are relatively small and exhibit little vertical variation. By 7.5 days the horizontally averaged flow is decelerated at all levels, with the most intense deceleration in three layers: 1) in the lower stratosphere, $11 \leq z \leq 14 \mathrm{~km}, 2)$ the upper troposphere $7 \leq z \leq 10 \mathrm{~km}$, and 3) near the surface. The layers of decelerated flow near the surface and in the lower stratosphere roughly coincide with regions of momentum flux divergence shown in Fig. 7. The decelerated layer in the upper troposphere, however, develops at heights where the vertical gradients in $M^{\prime}$ are very weak. In the next section, we will examine the momentum budget in each of these layers to gain deeper insight into the factors responsible for the deceleration. 




FIG. 8. The zonal velocity perturbation $u^{\prime}\left(\mathrm{m} \mathrm{s}^{-1}\right)$ in the $2-\mathrm{km}$ simulation as a function of height and time, horizontally averaged over the entire nested mesh.

\section{b. The momentum budget for finite-amplitude rotating flow}

To analyze the momentum budget, we begin with the momentum equation along the $x$ coordinate,

$$
\frac{\partial \rho u}{\partial t}+\nabla \cdot(\rho u \mathbf{v}+p \mathbf{i})-\rho f v=D_{u},
$$

where $\mathbf{v}$ is the $3 \mathrm{D}$ velocity vector, $\mathbf{i}$ is the unit vector parallel to the $x$ coordinate, and $D_{u}$ represents turbulent mixing. Let the ageostrophic wind $v_{\text {ag }}$ be defined such that

$$
\rho f v_{\mathrm{ag}}=\rho f v-\frac{\partial p}{\partial x}
$$

note that when this relationship is applied on small scales around the topography, the ageostrophic wind may not be a small correction to the geostrophic wind. Let angle brackets denote the integral over a volume $V$ defined by the cuboid $\left(x_{1}, x_{2}\right) \times\left(y_{1}, y_{2}\right) \times\left(z_{1}, z_{2}\right)$. Substituting (11) into (10) and integrating over $V$ yields

$\frac{\partial\langle\rho u\rangle}{\partial t}=-[\rho u u]_{x_{1}}^{x_{2}}-[\rho u v]_{y_{1}}^{y_{2}}-[\rho u w]_{z_{1}}^{z_{2}}+\left\langle f \rho v_{\mathrm{ag}}\right\rangle+\left\langle D_{u}\right\rangle$,

where the first three terms on the right-hand side are differences of the integrals of the advective fluxes of $\rho u$ across the cuboid faces perpendicular to the $x, y$, and $z$ axes, respectively: for example

$$
\begin{aligned}
{\left.[\rho u u]\right|_{x_{1}} ^{x_{2}}=} & \int_{y_{1}}^{y_{2}} \int_{z_{1}}^{z_{2}} \rho u u\left(x_{2}, y, z\right) d y d z \\
& -\int_{y_{1}}^{y_{2}} \int_{z_{1}}^{z_{2}} \rho u u\left(x_{1}, y, z\right) d y d z .
\end{aligned}
$$

If a budget volume, denoted $V_{s}$, extends to the surface, the cross-mountain pressure drag contributes to the momentum budget, but it may be absorbed into $\left\langle f \rho v_{\mathrm{ag}}\right\rangle_{s}$, where \langle\rangle$_{s}$ denotes the integral over $V_{s}$. To appreciate the role of the cross-mountain-pressure drag, note that if $\mathbf{n}$ is the outward directed unit normal vector, then by the divergence theorem

$$
\iiint_{V_{s}} \nabla \cdot(\rho u \mathbf{v}+p \mathbf{i}) d V_{s}=\iint_{A_{s}}(\rho u \mathbf{v}+p \mathbf{i}) \cdot \mathbf{n} d A_{s} .
$$

Integrating (10) over $V_{s}$, the surface integral contributions from the lateral sides and the top of $V_{s}$ are exactly as in (12). At the lower boundary, if $\mathbf{G}$ is a vector field,

$\int_{x_{1}}^{x_{2}} \int_{y_{1}}^{y_{2}} \mathbf{G} \cdot \mathbf{n} d A=\int_{x_{1}}^{x_{2}} \int_{y_{1}}^{y_{2}}\left[G_{(x)} \frac{\partial h}{\partial x}+G_{(y)} \frac{\partial h}{\partial y}-G_{(z)}\right] d y d x$,

where the differential element of surface area $d A$ satisfies

$$
d A=\left[\left(\frac{\partial h}{\partial x}\right)^{2}+\left(\frac{\partial h}{\partial y}\right)^{2}+1\right]^{1 / 2} d y d x .
$$

Since there are no advective fluxes across terrain at the lower boundary, (14) and (15) may be used to express the integral of (10) over $V_{s}$ as

$$
\begin{aligned}
\frac{\partial\langle\rho u\rangle_{s}}{\partial t}= & -\left.[\rho u u]\right|_{x_{1}} ^{x_{2}}-\left.[\rho u v]\right|_{y_{1}} ^{y_{2}}-\left.[\rho u w]\right|_{z_{2}} \\
& +\langle\rho \rho v\rangle_{s}-\left.[p]\right|_{x_{1}} ^{x_{2}}-\int_{x_{1}}^{x_{2}} \int_{y_{1}}^{y_{2}} p(x, y, h) \\
& \times \frac{\partial h}{\partial x} d y d x+\left\langle D_{u}\right\rangle_{s} .
\end{aligned}
$$

Substituting

$$
\begin{aligned}
\left\langle f \rho v_{\mathrm{ag}}\right\rangle_{s}= & \langle f \rho v-\nabla \cdot p \mathbf{i}\rangle_{s}=\langle f \rho v\rangle_{s}-\left.[p]\right|_{x_{1}} ^{x_{2}} \\
& -\int_{x_{1}}^{x_{2}} \int_{y_{1}}^{y_{2}} p(x, y, h) \frac{\partial h}{\partial x} d y d x
\end{aligned}
$$

into (17) yields 


$$
\begin{aligned}
\frac{\partial\langle\rho u\rangle_{s}}{\partial t}= & -\left.[\rho u u]\right|_{x_{1}} ^{x_{2}}-\left.[\rho u v]\right|_{y_{1}} ^{y_{2}}-\left.[\rho u w]\right|_{z_{2}} \\
& +\left\langle f \rho v_{\mathrm{ag}}\right\rangle_{s}+\left\langle D_{u}\right\rangle_{s},
\end{aligned}
$$

which is identical to (12) except that there is no contribution from the vertical momentum flux $\rho u w$ at the lower boundary.

\section{c. Layer-integrated perturbation momentum budgets}

The mountain-induced changes in the momentum balance in each layer are computed by taking the difference between the budgets for the mountain and no-mountain simulations. Neglecting the trivial mountain-induced differences in the density, this immediately converts the terms in (12), (17), and (19) that are linear in the unknowns to corresponding expressions for the perturbations. Letting the subscripts $m$ and $n$ denote fields from the mountain and no-mountain simulations, and recalling that $u^{\prime}=u_{m}-u_{n}$, the horizontal and vertical momentum flux terms, may be decomposed as follows:

$$
\begin{aligned}
& (\rho u u)^{\prime}=\rho u^{\prime} u^{\prime}+2 \rho u_{n} u^{\prime}, \\
& (\rho u v)^{\prime}=\rho u^{\prime} v^{\prime}+\rho u_{n} v^{\prime}+\rho u^{\prime} v_{n}, \\
& (\rho u w)^{\prime}=\rho u^{\prime} w^{\prime}+\rho u_{n} w^{\prime}+\rho u^{\prime} w_{n} .
\end{aligned}
$$

In the above, the first terms on the right-hand sides, which are products of perturbations, represent fluxes carried by mountain waves and other mesoscale terraininduced circulations. When integrated over the face of a budget volume, the remaining terms on the right-hand sides, which are first order in the perturbations, are primarily generated by small terrain-induced changes to the large-scale flow. Because the mountain-wave scales are much shorter than those of the large-scale nomountain fields, their contributions to expressions like

$$
\int_{x_{1}}^{x_{2}} \int_{z_{1}}^{z_{2}} \rho u_{n}\left(x, y_{2}, z\right) v^{\prime}\left(x, y_{2}, z\right) d x d z
$$

integrate approximately to zero in comparison with the integral of the longer wavelength components of $v^{\prime}$ arising from the difference between the mountain and no-mountain synoptic-scale fields. (An example of the perturbations that give rise to a large $2 \rho u_{n} u^{\prime}$ flux will be described in connection with Fig. 16.) In the following analysis, the contributions from all the firstorder terms in (20)-(22) are lumped together; they turn out to be dominated by the horizontal fluxes. In addition to having little impact compared to the horizontal first-order fluxes, the horizontal averages of $\rho u_{n} w^{\prime}+\rho u^{\prime} w_{n}$ are also much smaller than the horizontal average of $\rho u^{\prime} w^{\prime}$.

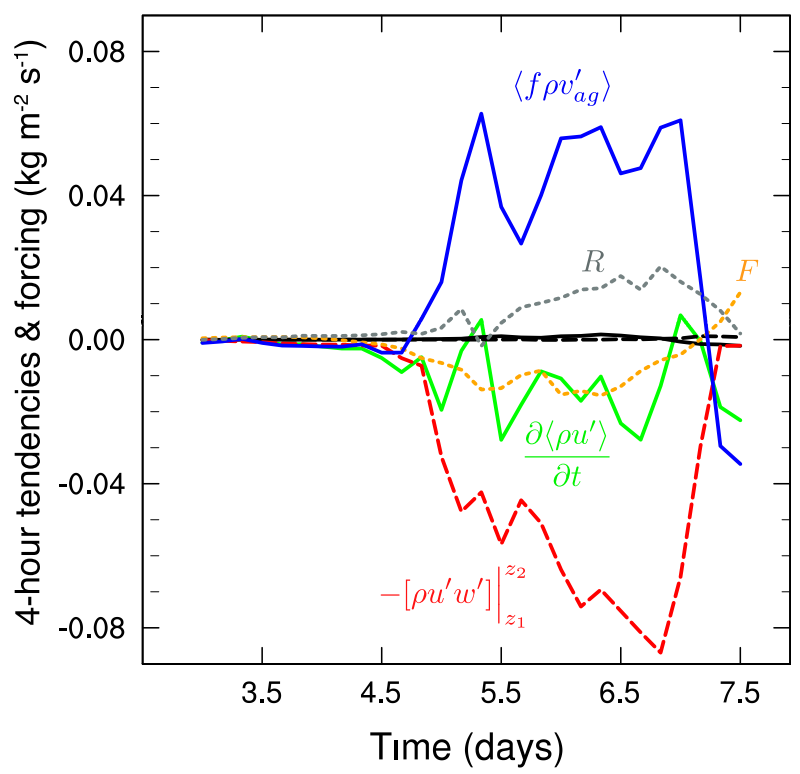

FIG. 9. Evolution of the terms in the perturbation momentum budget from (24) for the layer between 12 and $14 \mathrm{~km}$ : perturbation ageostrophic Coriolis forcing (blue), vertical momentum flux divergence $-\left.\left[\rho u^{\prime} w^{\prime}\right]\right|_{z_{1}} ^{z_{2}}$ (dashed red), $-\left.\left[\rho u^{\prime} u^{\prime}\right]\right|_{x_{1}} ^{x_{2}}$ (solid black), $-\left.\left[\rho u^{\prime} v^{\prime}\right]\right|_{y_{1}} ^{y_{2}}$ (long-dashed black), perturbation zonal momentum tendency (green), the sum of the flux divergences that are first order in the perturbations $F$ ( $\tan$ dashed line), and residual $R$ (gray short dashed).

The average deceleration in a layer above the surface is governed by difference between the mountain- and no-mountain momentum budgets in (12), which may be written as

$$
\begin{aligned}
\frac{\partial\left\langle\rho u^{\prime}\right\rangle}{\partial t}= & -\left.\left[\rho u^{\prime} u^{\prime}\right]\right|_{x_{1}} ^{x_{2}}-\left.\left[\rho u^{\prime} v^{\prime}\right]\right|_{y_{1}} ^{y_{2}}-\left.\left[\rho u^{\prime} w^{\prime}\right]\right|_{z_{1}} ^{z_{2}} \\
& +\left\langle f \rho v_{\mathrm{ag}}^{\prime}\right\rangle+F+R,
\end{aligned}
$$

where $F$ denotes the divergence of the fluxes that are first order in the perturbations,

$$
\begin{aligned}
F= & -\left.\left[2 \rho u_{n} u^{\prime}\right]\right|_{x_{1}} ^{x_{2}}-\left.\left[\rho\left(u^{\prime} v_{n}+u_{n} v^{\prime}\right)\right]\right|_{y_{1}} ^{y_{2}} \\
& -\left.\left[\rho\left(u^{\prime} w_{n}+u_{n} w^{\prime}\right)\right]\right|_{z_{1}} ^{z_{2}},
\end{aligned}
$$

and $R$ is the residual, which includes any contributions from mixing across the sides of the budget volume and the integrated effects of numerical smoothing. If all terrain-induced perturbations vanish at the lateral boundaries of the budget volume and there are no turbulent fluxes across the top and bottom faces, and if the vertical momentum flux is dominated by the mountain waves, (12) reduces to

$$
\left.\left[\rho u^{\prime} w^{\prime}\right]\right|_{z_{1}} ^{z_{2}}=-\frac{\partial\left\langle\rho u^{\prime}\right\rangle}{\partial t}+\left\langle f \rho v_{\mathrm{ag}}^{\prime}\right\rangle
$$



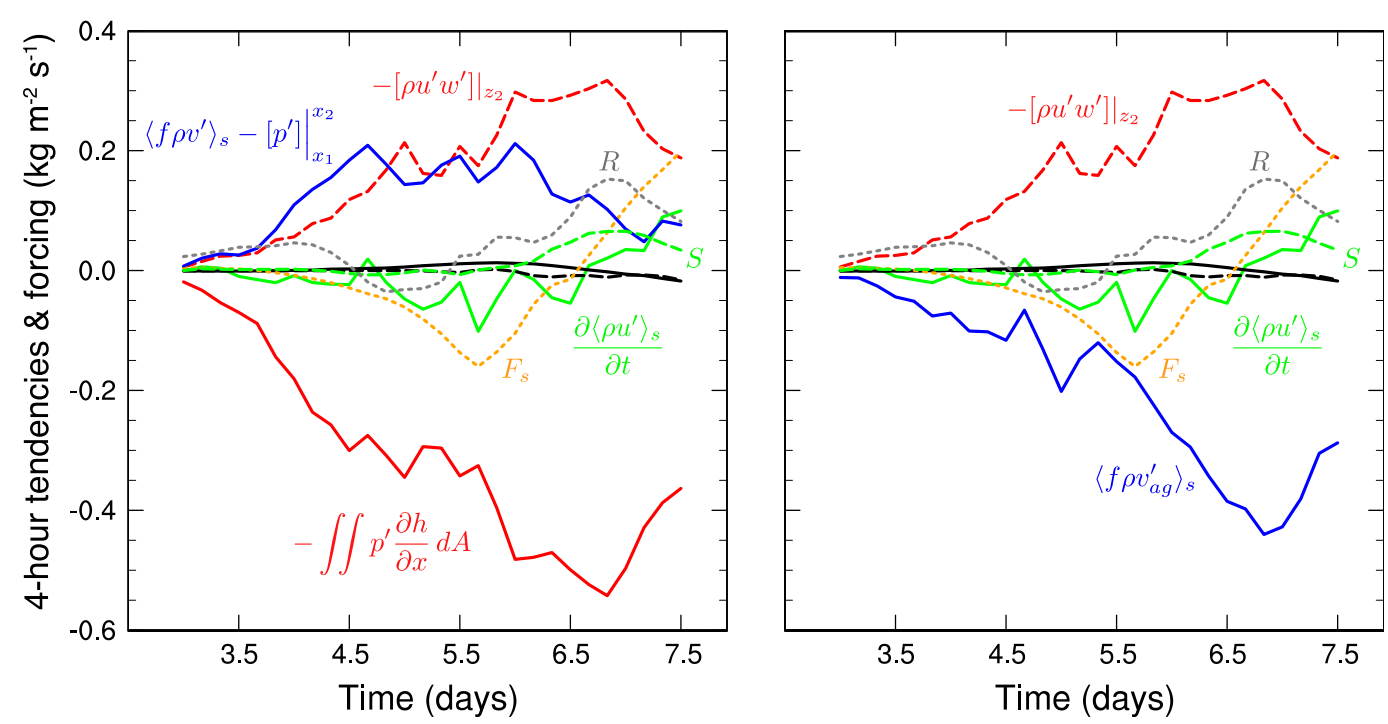

FIG. 10. Evolution of the terms in the perturbation momentum budget for the layer between the surface and $2 \mathrm{~km}$ : (left) from (27) and (right) from (29). Both panels include the vertical momentum flux at $2 \mathrm{~km}-\left.\left[\rho u^{\prime} w^{\prime}\right]\right|_{z_{2}}$ (dashed red), $-\left.\left[\rho u^{\prime} u^{\prime}\right]\right|_{x_{1}} ^{x_{2}}$ (solid black), $-\left.\left[\rho u^{\prime} v^{\prime}\right]\right|_{y_{1}} ^{y_{2}}$ (long-dashed black), perturbation zonal momentum tendency (green), surface stress (dashed green), the sum of the flux divergences that are first order in the perturbations $F$ ( $\tan$ dashed line), and the residual $R$ (gray dashed). Plotted only in the left panel are the pressure drag (solid red) and the perturbation Coriolis forcing plus the pressure difference across the domain (blue), which are replaced in the right panel by their sum, the perturbation ageostrophic Coriolis forcing (blue).

implying the vertical momentum flux divergence is balanced by the sum of the domain integrated crossmountain deceleration and Coriolis forces associated with the perturbation meridional ageostrophic motion.

The individual terms in (24), evaluated for the $12 \leq z \leq 14-\mathrm{km}$ layer, are plotted as a function of time in Fig. 9. To make the dimensional budget values easier to interpret, all the values shown in Figs. 9-11 are divided by the volume of the layer in question and multiplied by $4 \mathrm{~h}$; as such they represent average tendencies and forcings over a 4-h period. The dominant balance in the $12 \leq z \leq 14-\mathrm{km}$ layer is clearly between the vertical momentum flux divergence associated with the mountain waves $\left.\left[\rho u^{\prime} w^{\prime}\right]\right|_{z_{1}} ^{z_{2}}$ and $\left\langle f \rho v_{\mathrm{ag}}^{\prime}\right\rangle$. The mean-flow deceleration in this layer is roughly equal to the sum of these two terms, as envisioned in (26), but this is somewhat serendipitous because the contributions from the other nonnegligible terms, the residual $R$ and the divergence of the first-order fluxes $F$, largely balance. The impact of the zonal and meridional momentum fluxes $\left(\rho u^{\prime} u^{\prime}\right.$ and $\left.\rho u^{\prime} v^{\prime}\right)$ carried by the mountain waves is very small.

We now consider the perturbation momentum budget in the layer between the surface and $z=2 \mathrm{~km}$. Taking the difference between the mountain- and no-mountain simulations, (17) becomes

$$
\begin{aligned}
\frac{\partial\left\langle\rho u^{\prime}\right\rangle_{s}}{\partial t}= & -\left.\left[\rho u^{\prime} u^{\prime}\right]\right|_{x_{1}} ^{x_{2}}-\left.\left[\rho u^{\prime} v^{\prime}\right]\right|_{y_{1}} ^{y_{2}}-\left.\left[\rho u^{\prime} w^{\prime}\right]\right|_{z_{2}} \\
& +\left\langle f \rho v^{\prime}\right\rangle_{s}-\left.\left[p^{\prime}\right]\right|_{x_{1}} ^{x_{2}}-\int_{x_{1}}^{x_{2}} \int_{y_{1}}^{y_{2}} p^{\prime}(x, y, h) \frac{\partial h}{\partial x} d y d x \\
& +F_{s}+R+S
\end{aligned}
$$

where $R$ is once again the residual, $S$ is the perturbation surface stress (which has been broken out of the turbulent mixing terms that are otherwise contained in $R$ ), and

$$
\begin{aligned}
F_{s}= & -\left.\left[2 \rho u_{n} u^{\prime}\right]\right|_{x_{1}} ^{x_{2}}-\left.\left[\rho\left(u^{\prime} v_{n}+u_{n} v^{\prime}\right)\right]\right|_{y_{1}} ^{y_{2}} \\
& -\left.\left[\rho\left(u^{\prime} w_{n}+u_{n} w^{\prime}\right)\right]\right|_{z_{2}} .
\end{aligned}
$$

The evolution of the terms in (27) are shown in Fig. 10a. The pressure drag is decelerative, and is roughly twice the magnitude of the next two largest terms, which are the vertical mountain-wave momentum flux through the 2-km level and the sum of perturbations to the Coriolis force acting on the perturbation meridional wind and the upstream-downstream pressure difference.

The budget is much simpler when written in terms of the Coriolis force acting on the perturbation ageostrophic meridional wind following (19), which when expressed as the difference between mountain and no-mountain simulations becomes 


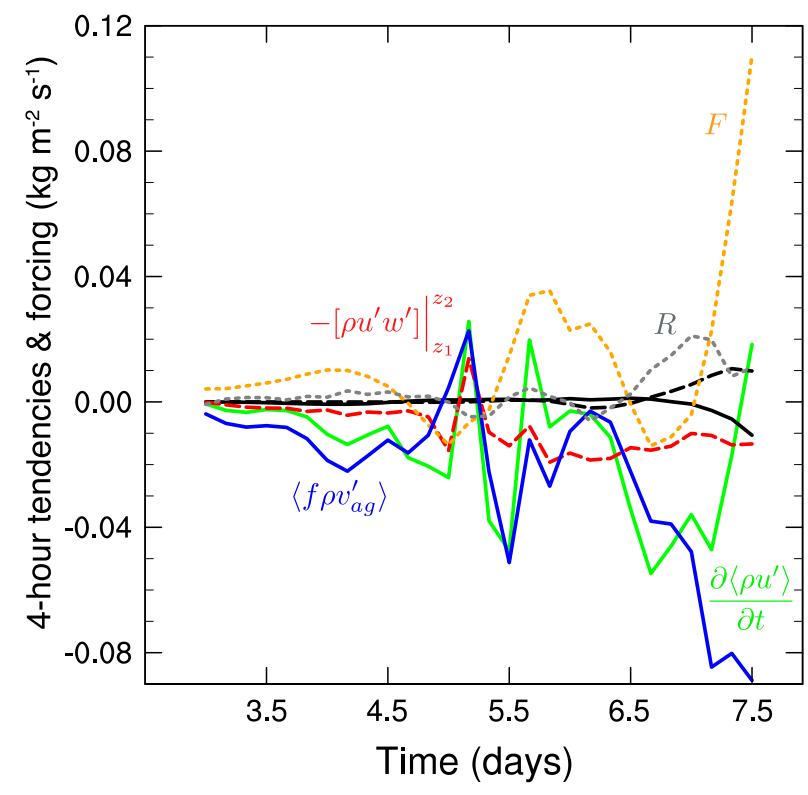

FIG. 11. As in Fig. 9, but for the layer between 7 and $10 \mathrm{~km}$.

$$
\begin{aligned}
\frac{\partial\left\langle\rho u^{\prime}\right\rangle_{s}}{\partial t}= & -\left.\left[\rho u^{\prime} u^{\prime}\right]\right|_{x_{1}} ^{x_{2}}-\left.\left[\rho u^{\prime} v^{\prime}\right]\right|_{y_{1}} ^{y_{2}}-\left.\left[\rho u^{\prime} w^{\prime}\right]\right|_{z_{2}} \\
& +\left\langle\rho \rho v_{\mathrm{ag}}^{\prime}\right\rangle_{s}+F_{s}+R+S .
\end{aligned}
$$

If all terrain-induced perturbations vanish at the lateral boundaries of the budget volume and there are no turbulent fluxes across the top of the volume and surface stress is neglected, and if the vertical momentum flux is dominated by the mountain waves, (29) reduces to

$$
\left.\left[\rho u^{\prime} w^{\prime}\right]\right|_{z_{2}}=-\frac{\partial\left\langle\rho u^{\prime}\right\rangle_{s}}{\partial t}+\left\langle f \rho v_{\mathrm{ag}}^{\prime}\right\rangle_{s}
$$

implying the vertical mountain-wave momentum flux through $z=z_{2}$ is equal to the sum of the domainintegrated cross-mountain deceleration and the Coriolis force associated with $v_{\mathrm{ag}}^{\prime}$. If the flow is also steady, (30) implies that downward momentum fluxes through a horizontal plane are balanced by the underlying perturbation equatorward ageostrophic motion.

The evolution of the perturbation momentum budget as characterized by (29) is plotted for the volume between the surface and $2 \mathrm{~km}$ in Fig. 10b. Analogous to the situation in the lower stratosphere, the dominant balance is between the momentum fluxed vertically into the layer and $\left\langle f \rho v_{\mathrm{ag}}^{\prime}\right\rangle_{s}$. Turning to the remaining smaller, but nonnegligible terms, the residual reaches its maximum value around day 6.7 as a result of lowlevel wave breaking creating mixing across the 2-km level (Fig. 2c). The first-order fluxes $F_{s}$ reach a negative extrema around 5.5 days as $u^{\prime}$ perturbations develop at the upstream boundary; these subsequently decay, and a positive $F_{s}$ maximum occurs at 7.5 days when $u^{\prime}$ perturbations grow large at the downstream boundary. Over most of the simulation, the volume-integrated momentum tendency is negative, although it becomes positive after 6.5 days, when, except for the pressure drag (and therefore $\left\langle\rho \rho v_{\mathrm{ag}}^{\prime}\right\rangle_{s}$ ), all the nonnegligible contributions to the budget are accelerative.

The dominant balance in the momentum budget for the strongly decelerated layer $(7 \leq z \leq 10 \mathrm{~km})$ is quite different from that in lower stratosphere. As shown in Fig. 11, the vertical momentum flux divergence across this layer is rather small (which is consistent with Fig. 7). Until 7 days, the flow deceleration is primarily balanced by the Coriolis force acting on perturbation ageostrophic winds blowing toward the south (the blue and green curves follow each other). After 7 days, $\left\langle f \rho v_{\mathrm{ag}}^{\prime}\right\rangle$ is still a decelerative forcing, but $F$ starts to become large and positive because of the contribution to $2 \rho u_{n} u^{\prime}$ at the downstream boundary. The feature responsible for the large signal in $2 \rho u_{n} u^{\prime}$ will be discussed in the next section. In summary, although Fig. 8 shows the $7 \leq z \leq 10-\mathrm{km}$ layer is the portion of the domain that undergoes the greatest horizontally averaged flow deceleration, that deceleration is not primarily produced by vertical momentum flux divergence across the layer.

\section{Local flow response}

We now examine the local flow response in each of the three layers considered in the previous section. Figure 12 shows isobars for the 2-km-mountain case, along with perturbation velocity vectors $\left(u^{\prime}, v^{\prime}\right)$ and the perturbation Ertel potential vorticity $\left(\mathrm{PV}^{\prime}\right)$ at $z=13 \mathrm{~km}$. Wave breaking at this level begins around 5 days, and by 5.5 days produces a complex pattern of positive and negative $\mathrm{PV}^{\prime}$ in the immediate lee of the ridge. Despite the north-south uniformity of the underlying ridge, the chaotic distribution of these PV anomalies is reminiscent of that obtained in high-resolution case-study simulations of flow over the Southern Alps (Kruse et al. 2016). In particular, there is little tendency to produce a pair of intense opposite-signed PV banners at each end of the wave breaking region as envisioned in Schär and Durran (1997), who argued that wave breaking aloft would produce PV banners similar to those generated at low levels by flow separation on the flanks of high topography (Schär et al. 2003). Nevertheless, larger-scale structures become apparent as the initial PV anomalies are carried downstream, with negative anomalies dominating on the left side of the large-scale jet, and positives on the right side (Figs. 12c,d) in a pattern similar to the simpler barotropic jet case 

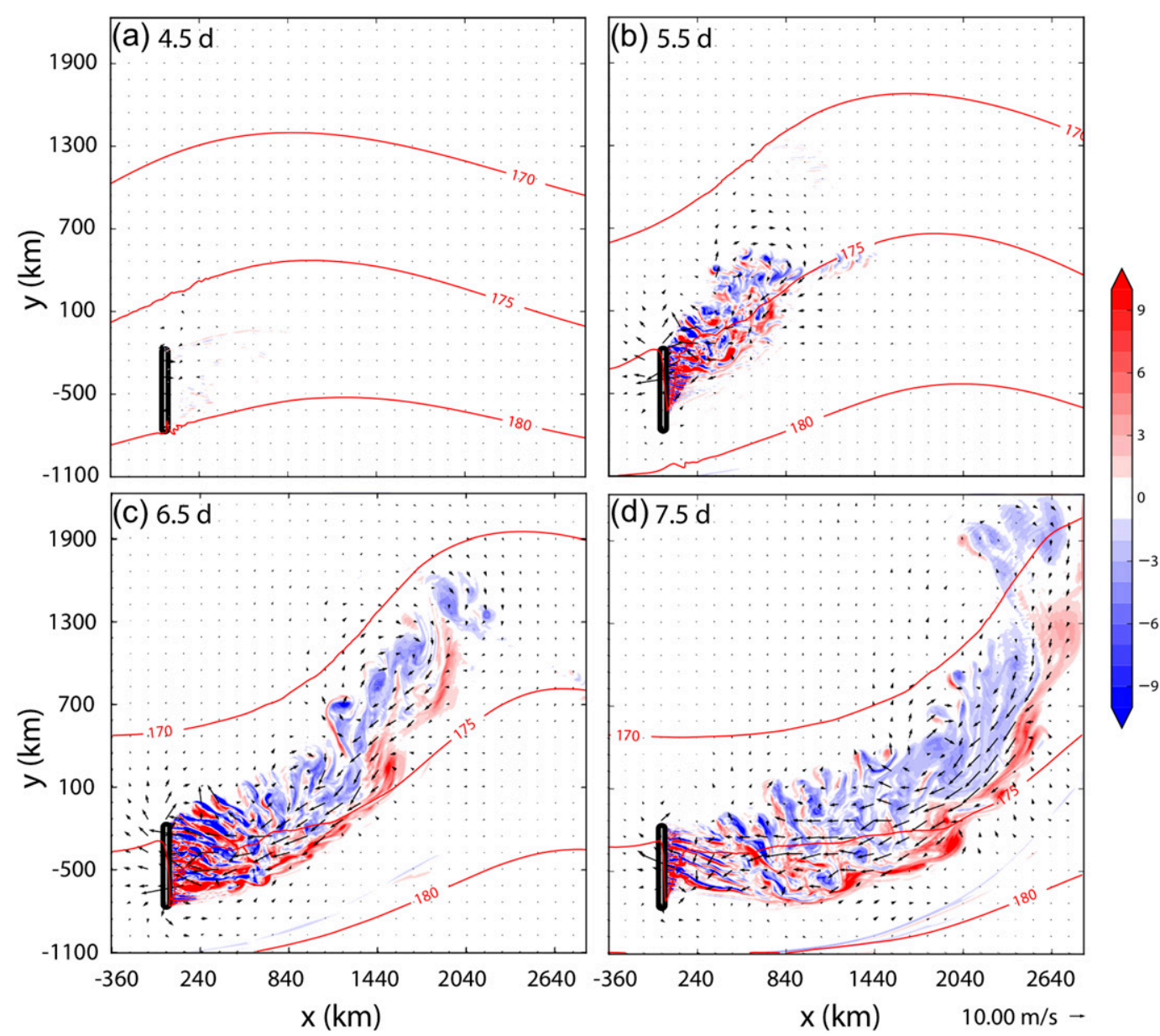

FIG. 12. Perturbation Ertel potential vorticity at $z=13 \mathrm{~km}$ [color fill; PVU (1 PVU $\left.=10^{-6} \mathrm{~K} \mathrm{~kg}^{-1} \mathrm{~m}^{2} \mathrm{~s}^{-1}\right)$ ], perturbation horizontal velocity vectors and pressure contours (red lines; hPa) at (a) 4.5, (b) 5.5, (c) 6.5, and (d) 7.5 days.

considered in Chen et al. (2007). By 7.5 days, significant velocity perturbations have developed along the jet axis that appear to be at least qualitatively in balance with the downstream PV' field. These perturbation velocities exceed $15 \mathrm{~m} \mathrm{~s}^{-1}$ at 7.5 days and oppose the winds along the core of the jet (Fig. 13a). The total horizontal velocity vectors and wind speeds from the 2-km-mountain simulation are plotted in Fig. 13b, which shows the winds along the center of the jet downstream of the mountain drop to less than $20 \mathrm{~m} \mathrm{~s}^{-1}$. Comparing Fig. 13b with Fig. 14, which shows the corresponding wind speeds in the no-mountain case; it is apparent that gravity wave breaking has produced a dramatic weakening of the jet maximum in the lower stratosphere.

Perturbation horizontal velocity vectors and wind speeds are plotted with isobars at $z=1 \mathrm{~km}$ and 7.5 days in Fig. 15a. The perturbation velocities form a distinct cyclonic vortex in the lee of the ridge, with reversed winds in large patches on the northern side of the vortex exceeding $15 \mathrm{~m} \mathrm{~s}^{-1}$. The vortex in Fig. 15a does not appear to be in balance with the $\mathrm{PV}^{\prime}$ field behind the mountain (not shown), perhaps because, in contrast to the situation in the lower stratosphere, those PV anomalies have not had time to organize as larger-scale structures as they drift downstream from the region in which they are generated. Although the perturbation velocities form a distinct vortex, the total velocities form a patch of almost stagnant flow downstream of the ridge (Fig. 15b).

Finally, let us consider the local response in the upper troposphere. Figure 16a shows the perturbation horizontal velocity vectors and wind speeds, together with isobars, at $z=9 \mathrm{~km}$ and 7.5 days. The maximum wind speed perturbations at this level are substantially weaker than those near the surface or in the lower stratosphere. Two patches of deceleration appear in the lee of the ridge, and the similarity of the perturbation velocity vectors around each of these patches suggests 

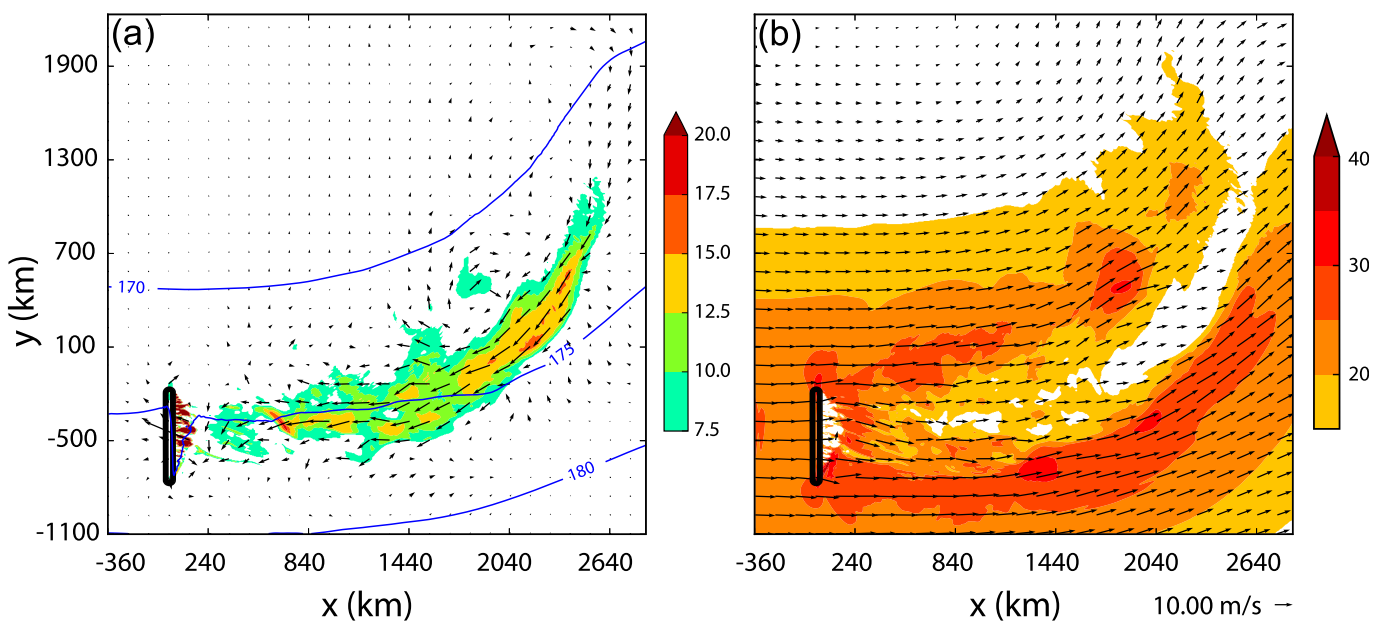

FIG. 13. Horizontal winds at 7.5 days and $z=13 \mathrm{~km}$ : (a) perturbation wind vectors $\left(u^{\prime}, v^{\prime}\right)$, speeds (color fill; $\mathrm{m} \mathrm{s}^{-1}$ ), and pressure contours (blue lines; $\mathrm{hPa}$ ); (b) total wind vectors and speeds (color fill; $\mathrm{m} \mathrm{s}^{-1}$ ) for the 2-km-mountain case.

a wavelike response, perhaps an inertial gravity wave. As shown in Fig. 16b, the terrain-induced deceleration has much less effect on the total velocity along the jet axis than that which is produced in the lower stratosphere (Fig. 13b). Thus, the local terrain-induced response is weakest in the same layer that exhibits the strongest domain-averaged deceleration! The strong domain-averaged signal is produced by widespread small-amplitude decelerations; a similar result was obtained by Durran (1995) in 2D simulations without the Coriolis force.

As noted during the discussion of momentum budgets averaged over the $7 \leq z \leq 10$-km layer, the terms $F$, which are first order in the mountain-no-mountain perturbations, make a large positive contribution to the momentum balance at 7.5 days, and this contribution is dominated by the divergence of the flux $2 \rho u_{n} u^{\prime}$. The motions responsible for this flux are easily seen in Fig. 16a over the interval $0 \leq y \leq 700 \mathrm{~km}$ along the eastern boundary. Here $u^{\prime}$ is negative; $u_{n}$, which can be inferred from the isobars, is strongly positive, and, because there is little signal in $u^{\prime}$ at the western boundary, $-\left.\left[2 \rho u_{n} u^{\prime}\right]\right|_{x_{1}} ^{x_{2}}$ is also positive. The $u^{\prime}$ field is roughly in quasigeostrophic balance with an elongated northsouth-oriented anticyclonic PV perturbation (not shown) centered near $(x, y)=(2640,1300) \mathrm{km}$. That PV perturbation resulted from a slight shift in the position of the jet and tropopause boundary between the mountain and no-mountain simulations. Such a shift produces strong PV perturbations because the 9-km level is in the troposphere on the warm side of the jet, and in the high-static-stability stratosphere on the cold side. This example also illustrates the way that the first-order flux terms in (20)-(22) are primarily produced by shifts in the synoptic-scale flow, and not directly by the mountain waves. If the fine-mesh budget domain extended farther downstream, this contribution to $F$ would likely disappear, which would tend to make the deceleration in the $7 \leq z \leq 10$-km layer even stronger.

Smith et al. (2008) analyzed the background environmental winds sampled using a race-track flight pattern during six mountain-wave events over the Sierra Nevada. These flights were below the level of likely wave breaking and showed the environmental winds to

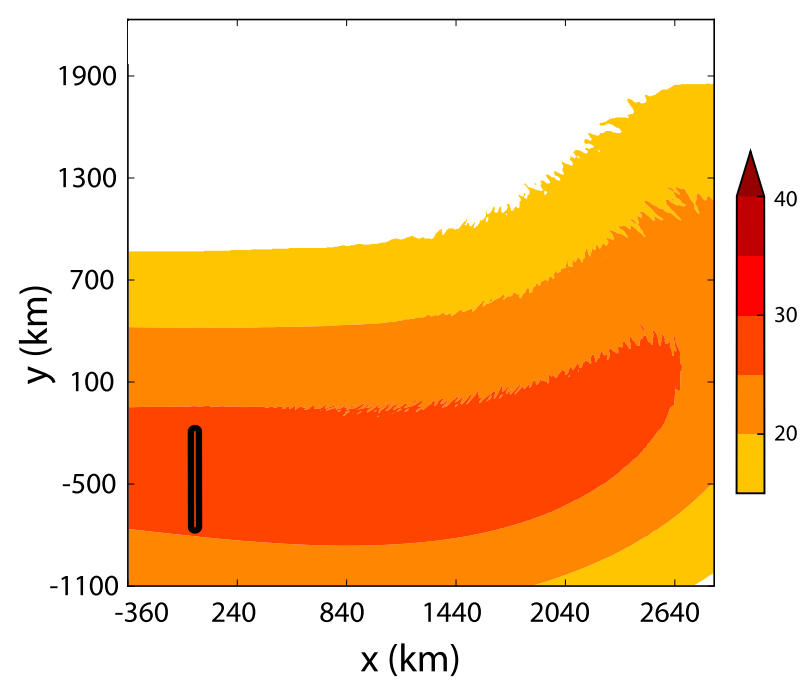

FIG. 14. Total horizontal wind speed $\left(\mathrm{m} \mathrm{s}^{-1}\right)$ for the no-mountain case at 7.5 days and $z=13 \mathrm{~km}$. Although there is no mountain, its position in the corresponding 2-km-mountain case is shown for reference. 

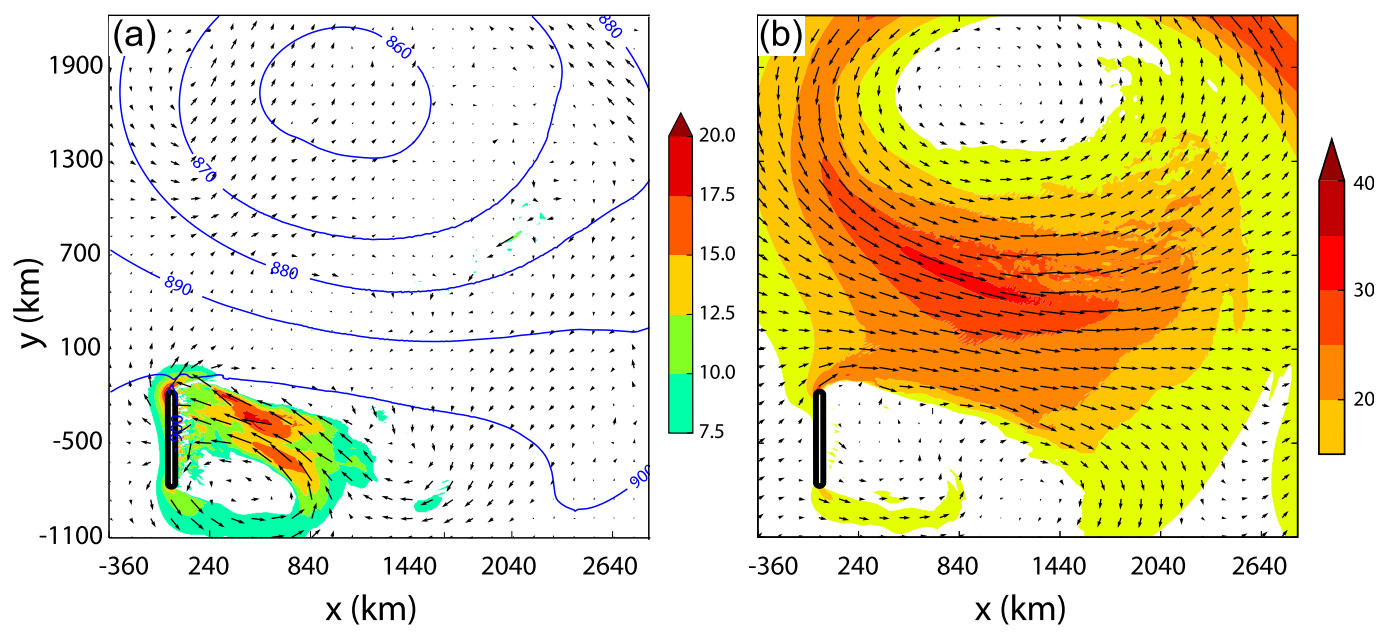

FIG. 15. As in Fig. 13, but at $z=1 \mathrm{~km}$.

be systematically subgeostrophic, which they attributed to gradient wind effects in the large-scale flow. Given our results in both Figs. 11 and 16, another explanation might be that the Coriolis force acting on ageostrophic circulations forced by wave breaking aloft helped produce those observed subgeostrophic winds.

\section{Conclusions}

We have numerically simulated an idealized cyclone growing in a baroclinically unstable jet as it crosses an isolated $3 \mathrm{D}$ ridge. One set of experiments using a 500-m-high ridge produced weakly nonlinear waves, while another set, using a 2-km-high ridge, produced strongly nonlinear waves and flow blocking. The wave response, itself, was previously discussed in MD17. Here the focus is on the vertical momentum fluxes, their relation to the cross-mountain pressure drag, and their influence on the large-scale flow.

The vertical momentum flux just above mountain top is closely related to pressure drag for the 500-m mountain, but is much weaker than the pressure drag in the 2 -km-mountain case. The difference in the case with the higher mountain is largely due to low-level wave breaking and flow blocking. The drag and momentum fluxes from steady-forcing simulations representative of the large-scale flow crossing the mountain at days $4.5,5.5,6.5$, and 7.5 greatly underestimate the strength of the response at the corresponding times in the evolving-flow 2-km-mountain case. The dependence of the vertical momentum flux on the flow evolution poses a potential complication in the formulation of accurate gravity wave drag parameterizations, which are typically based entirely on the instantaneous properties


FIG. 16. As in Fig. 13, but at $z=9 \mathrm{~km}$. 
of the cross-mountain flow. Over the 500-m mountain, the steady-forcing solution also greatly underestimates the fluxes and drag at 6.5 days, as the cross-mountain winds begin to decrease, but for this lower mountain, the steady and evolving large-scale flows generate similar results at the other times.

The interactions between the terrain and the largescale flow are much stronger in the 2-km-high-mountain simulation, which we used for further studies of the wave-mean flow interaction. The horizontally averaged budget for the perturbation momentum, evaluated from the difference between corresponding fields in mountain and no-mountain simulations, shows vertical momentum flux divergence occurring in layers near the surface and in response to wave breaking in the lower stratosphere. By the end of the simulation, horizontally averaged flow deceleration occurs in both of those layers, but is even stronger in the upper troposphere where the vertical gradients in the momentum flux are minimal. Theoretical analysis of the layer-averaged perturbation momentum budget shows that-provided the terraininduced perturbations are negligible at the boundaries - the mean-flow deceleration would be balanced by the sum of the vertical momentum flux divergence and the Coriolis force acting on the layer-averaged ageostrophic wind [see (26)]. If the budget volume extends to the surface, the cross-mountain pressure drag may be absorbed into contribution from the Coriolis force acting on the layer-averaged ageostrophic wind [see (30)]. These are indeed the most important terms in the budgets for the terrain-induced perturbations computed from the numerical simulations in a layer in the lower stratosphere and in the volume between $2 \mathrm{~km}$ and the surface.

In the upper troposphere, the contribution from the vertical momentum flux divergence is small, and the simulated flow decelerates in response to Coriolis forces acting on the ageostrophic wind. In the context of quasigeostrophic theory, ageostrophic circulations arise to restore thermal wind balance. The precise nature of the ageostrophic circulations responsible for the flow deceleration in the upper troposphere are the subject of continuing research. Near the end of the simulation, an $x$-component momentum flux that is first order in the mountain-no-mountain perturbations, $2 u_{n} u^{\prime}$ also makes an important accelerative contribution to the perturbation momentum budget in this layer. In contrast to those fluxes that are second-order in the perturbations, which are the momentum fluxes carried by the mountain waves themselves, the first-order fluxes arise from differences between the large-scale circulations in the mountain and no-mountain simulations, and are a manifestation of the large-scale response to the topography. The flux $2 u_{n} u^{\prime}$ becomes significant at the downstream boundary because of a shift in the axis of the upper-level jet, and would be greatly reduced if the budget domain extended farther downstream.

The local response of the large-scale flow to the terrain-induced perturbations varies with altitude. In the lower stratosphere, wave breaking generates a very strong reduction in the winds at the core of the jet. Near the surface, flow blocking and wave breaking produce a large region of stagnant flow behind the mountain that looks like a cyclonic vortex in the perturbation velocity field. The local deceleration in the upper troposphere moderately reduces the wind speed in two regions along the jet in a wavelike pattern. Although, among these three regions, the upper troposphere experiences the greatest layer-averaged deceleration, the maximum local deceleration in this layer is weaker than those in the lower stratosphere and the surface. The strong layer-averaged deceleration in the upper troposphere is produced by widespread, small-amplitude decelerations.

Acknowledgments. The authors are grateful for very helpful comments from three anonymous reviewers. This research was funded by NSF Grant AGS-1545927. Author Menchaca was also supported by a National Science Foundation Graduate Research Fellowship. We would like to acknowledge high-performance computing support from Yellowstone (ark:/85065/d7wd3xhc) provided by NCARs Computational and Information Systems Laboratory, sponsored by the National Science Foundation.

\section{REFERENCES}

Bessemoulin, P., P. Bougeault, A. Genoves, A. Jansa Clar, and D. Puech, 1993: Mountain pressure drag during PYREX. Contrib. Atmos. Phys., 66, 305-325.

Bougeault, P., and Coauthors, 1993: The atmospheric momentum budget over a major mountain range: First results of the PYREX field program. Ann. Geophys., 11, 395-418.

- B. Benech, P. Bessemoulin, B. Carissimo, A. Jansa Clar, J. Pelon, M. Petitdidier, and E. Richard, 1997: PYREX: A summary of findings. Bull. Amer. Meteor. Soc., 78, 637650, https://doi.org/10.1175/1520-0477(1997)078<0637: $\mathrm{PASOF}>2.0 . \mathrm{CO} ; 2$.

Bretherton, F., 1969: Momentum transport by gravity waves. Quart. J. Roy. Meteor. Soc., 95, 213-243, https://doi.org/ 10.1002/qj.49709540402.

Chen, C.-C., D. R. Durran, and G. Hakim, 2005: Mountain-wave momentum flux in an evolving synoptic-scale flow. J. Atmos. Sci., 62, 3213-3231, https://doi.org/10.1175/JAS3543.1.

—, G. J. Hakim, and D. Durran, 2007: Transient mountain waves and their interaction with large scales. J. Atmos. Sci., 64, 23782400, https://doi.org/10.1175/JAS3972.1.

Davies, H. C., and P. D. Phillips, 1985: Mountain drag along the Gotthard section during ALPEX. J. Atmos. Sci., 42, 
2093-2109, https://doi.org/10.1175/1520-0469(1985)042<2093: MDATGS $>2.0 . \mathrm{CO} ; 2$.

Doyle, J., Q. Jiang, R. Smith, and V. Grubišić, 2011: Threedimensional characteristics of stratospheric mountain waves during T-REX. Mon. Wea. Rev., 139, 3-23, https://doi.org/ 10.1175/2010MWR3466.1.

Durran, D. R., 1992: Two-layer solutions to Long's equation for vertically propagating mountain waves: How good is linear theory? Quart. J. Roy. Meteor. Soc., 118, 415-433, https://doi.org/ 10.1002/qj.49711850502.

,1995: Do breaking mountain waves decelerate the local mean flow? J. Atmos. Sci., 52, 4010-4032, https://doi.org/10.1175/ 1520-0469(1995)052<4010:DBMWDT>2.0.CO;2.

Eliassen, A., and E. Palm, 1960: On the transfer of energy in stationary mountain waves. Geofys. Publ., 22, 1-23.

Epifanio, C. C., and D. R. Durran, 2001: Three-dimensional effects in high-drag-state flows over long ridges. J. Atmos. Sci., $\mathbf{5 8}$, 1051-1065, https://doi.org/10.1175/1520-0469(2001)058<1051: TDEIHD $>2.0 . \mathrm{CO} ; 2$.

Fritts, D., and Coauthors, 2016: The Deep Propagating Gravity Wave Experiment (DEEPWAVE): An airborne and groundbased exploration of gravity wave propagation and effects from their sources throughout the lower and middle atmosphere. Bull. Amer. Meteor. Soc., 97, 425-453, https://doi.org/ 10.1175/BAMS-D-14-00269.1.

Gill, A., 1982: Atmosphere-Ocean Dynamics. Academic Press, $662 \mathrm{pp}$.

Jones, W. L., 1967: Propagation of internal gravity waves in fluids with shear flow and rotation. J. Fluid Mech., 30, 439-448, https://doi.org/10.1017/S0022112067001521.

Kim, Y.-J., S. D. Eckermann, and H.-Y. Chun, 2003: An overview of the past, present and future of gravity-wave drag parametrization for numerical climate and weather prediction models. Atmos.-Ocean, 41, 65-98, https://doi.org/10.3137/ao.410105.

Kruse, C., R. Smith, and S. D. Eckermann, 2016: The midlatitude lower-stratospheric mountain wave "valve layer". J. Atmos. Sci., 73, 5081-5100, https://doi.org/10.1175/JAS-D-16-0173.1.

Lilly, D. K., 1972: Wave momentum flux-A GARP problem. Bull. Amer. Meteor. Soc., 53,17-23, https://doi.org/10.1175/ 1520-0477-53.1.17.

— mountain wave and its associated momentum flux and energy dissipation. J. Atmos. Sci., 30, 1135-1152, https://doi.org/ 10.1175/1520-0469(1973)030<1135:OOASMW>2.0.CO;2.

, J. M. Nicholls, P. J. Kennedy, J. B. Klemp, and R. M. Chervin, 1982: Aircraft measurements of wave momentum flux over the Colorado Rocky Mountains. Quart. J. Roy. Meteor. Soc., 108, 625-642, https://doi.org/10.1002/qj.49710845709.
Lott, F., and H. Teitelbaum, 1993a: Linear unsteady mountain waves. Tellus, 45A, 201-220, https://doi.org/10.3402/ tellusa.v45i3.14871.

— and - 1993b: Topographic waves generated by a transient wind. J. Atmos. Sci., 50, 2607-2624, https://doi.org/10.1175/ 1520-0469(1993)050<2607:TWGBAT >2.0.CO;2.

Menchaca, M. Q., and D. R. Durran, 2017: Mountain waves, downslope winds, and low-level blocking forced by a midlatitude cyclone encountering an isolated ridge. J. Atmos. Sci., 74, 617-639, https://doi.org/10.1175/JAS-D-16-0092.1.

Ólafsson, H., and P. Bougeault, 1997: The effect of rotation and surface friction on orographic drag. J. Atmos. Sci., $\mathbf{5 4}$ 193-210, https://doi.org/10.1175/1520-0469(1997)054<0193: TEORAS $>2.0 . \mathrm{CO} ; 2$.

Sawyer, J. S., 1959: The introduction of the effects of topography into methods of numerical forecasting. Quart. J. Roy. Meteor. Soc., 85, 31-43, https://doi.org/10.1002/qj.49708536304.

Schär, C., and D. Durran, 1997: Vortex formation and vortex schedding in continuously stratified flows past isolated topography. J. Atmos. Sci., 54, 534-554, https://doi.org/10.1175/ 1520-0469(1997)054<0534:VFAVSI >2.0.CO;2.

—, M. Sprenger, D. Lüthi, Q. F. Jiang, R. B. Smith, and R. Benoit, 2003: Structure and dynamics of an alpine potential-vorticity banner. Quart. J. Roy. Meteor. Soc., 129, 825-855, https://doi.org/ 10.1256/qj.02.47.

Smith, R. B., 1979a: The influence of the Earth's rotation on mountain wave drag. J. Atmos. Sci., 36, 177-180, https://doi.org/ 10.1175/1520-0469(1979)036<0177:TIOTER >2.0.CO;2. , 1979b: The influence of the mountains on the atmosphere. Advances in Geophysics, B. Saltzman, Ed., Vol. 21, Academic Press, 87-230.

— J. D. Doyle, Q. Jiang, and S. A. Smith, 2007: Alpine gravity waves: Lessons from MAP regarding mountain wave generation and breaking. Quart. J. Roy. Meteor. Soc., 133, 917-936, https://doi.org/10.1002/qj.103.

_ B. Woods, J. Jensen, W. Cooper, J. Doyle, Q. Jiang, and V. Grubišić, 2008: Mountain waves entering the stratosphere. J. Atmos. Sci., 65, 2543-2562, https://doi.org/10.1175/ 2007JAS2598.1.

Snyder, W. H., R. S. Thompson, R. E. Eskridge, R. E. Lawson, I. P. Castro, J. T. Lee, J. C. R. Hunt, and Y. Ogawa, 1985: The structure of strongly stratified flow over hills: Dividingstreamline concept. J. Fluid Mech., 152, 249-288, https:// doi.org/10.1017/S0022112085000684.

Wells, H., S. Webster, and A. Brown, 2005: The effect of rotation on the pressure drag force produced by flow around long mountain ridges. Quart. J. Roy. Meteor. Soc., 131, 1321-1338, https://doi.org/10.1256/qj.04.37. 